$\mathrm{IMSc} / 2004 / 04 / 16$

AEI-2004-028

\title{
The Bianchi IX model in Loop Quantum Cosmology
}

\author{
Martin Bojowald,,$*$ Ghanashyam Date, ${ }^{2, t}$ and Golam Mortuza Hossain ${ }^{2, t}$ \\ ${ }^{1}$ Max-Planck-Institut für Gravitationsphysik, Albert-Einstein-Institut, \\ Am Mühlenberg 1, D-14476 Golm, Germany \\ ${ }^{2}$ The Institute of Mathematical Sciences \\ CIT Campus, Chennai-600 113, INDIA.
}

\begin{abstract}
The Bianchi IX model has been used often to investigate the structure close to singularities of general relativity. Its classical chaos is expected to have, via the BKL scenario, implications even for the approach to general inhomogeneous singularities. Thus, it is a popular model to test consequences of modifications to general relativity suggested by quantum theories of gravity. This paper presents a detailed proof that modifications coming from loop quantum gravity lead to a non-chaotic effective behavior. The way this is realized, independently of quantization ambiguities, suggests a new look at initial and final singularities.
\end{abstract}

PACS numbers: 0460P, 0460K, 9880H

*Electronic address: mabo@aei.mpg.de

${ }^{\dagger}$ Electronic address: shyam@imsc.res.in

${ }^{\ddagger}$ Electronic address: golam@imsc.res.in 


\section{INTRODUCTION}

It is well known that according to the singularity theorems [1], the space-time describing the backward evolution of an expanding universe is necessarily singular in the sense of geodesic incompleteness. The generality of singularity theorems also prevents them from giving any information about the nature of such a singularity for example in terms of curvature invariants. By contrast, the BKL approach to the issue of singularities asks [2]: In a neighborhood of a presumed singularity, is there a general solution of the Einstein equation such that at least some curvature invariants diverge? In this formulation of the question, one also obtains information about the possible nature of the presumed singularity described in terms of the approach to the singularity. The conclusion of the long and detailed analysis is summarized in the BKL scenario: Generically, as the singularity is approached, the spatial geometry can be viewed as a collection of small patches each of which evolves essentially independently of the others according to the Bianchi IX evolution (for recent numerical evidence see [3]). The Bianchi IX evolution towards its singularity is described by an infinite succession of Kasner evolutions (two directions contracting while third one expanding) punctuated by permutations of the expanding/contracting directions as well as possible rotations of the three directions themselves. The qualitative analysis of these permutations and rotations ('oscillations') is done very conveniently in terms of a billiard ball bouncing off moving walls and has been analyzed for a possible chaotic behavior [4].

However, it has been well recognized that the conclusion of ad infinitum oscillations of the BKL singularity, a consequence of unbounded growth of the spatial curvature, cannot be trusted very close to the singularity where the classical Einstein equations themselves are expected to break down. Presumably the equations will be superceeded by some quantum extensions. In the absence of any specific and detailed enough quantum theory the questions of the fate of the classical singularity vis a vis the behavior of space-time near such a region, could not have been addressed. The focus therefore has been to include qualitatively expected quantum modification. For example, in the Kaluza-Klein picture and more recently the stringy picture, the minimal expected modification is the matter content - notably the dilaton and p-form fields. Higher derivative corrections in the effective action are also expected. Another qualitative implication of string theory namely the brane world scenarios is also a candidate to study implications of modified Einstein equations. All of these have 
been explored with varying conclusions regarding the BKL behavior, e.g. [5, 6]. However, they still contain the singularity and hence all these modified equations must break down near the singularity raising questions about the validity of the conclusion.

In recent times this situation has changed, particularly in the cosmological context. Within the loop quantum gravity approach [7], we do have the detailed framework of LQC 8] to deduce the nature of quantum effects. Due to the underlying quantum geometry 9, 10, 11, 12], the internal time (volume) evolution becomes a difference equation [13, 14] and one also has a quantization of the inverse scale factor with a bounded spectrum [15]. Thus the classically indicated singularity is removed i.e. the evolution extends to 'negative' (internal) time and the spatial curvatures remain finite [16, 17, 18, 19]. The equations also admit a continuum approximation thereby recovering the classical continuum geometry for larger volumes [20, 21].

It is now meaningful to ask if and how the BKL behavior is modified. There is in fact a hint of what may be expected. For larger volumes, one can trust the classical picture. Then, in the general inhomogeneous cosmological context, one can approximate smaller patches of the spatial geometry by the homogeneous Bianchi IX models. As the volume is decreased, the patches have to be made smaller to sustain the approximation. If the BKL behavior were to continue for these individual patches, then the fragmentation into smaller patches must continue ad infinitum. But the underlying discrete structure cannot support such infinite fragmentation. Therefore, the quantum geometry which is responsible for singularity removal, must also ensure that Bianchi IX behavior may at the most have finitely many oscillations. This is in fact a self consistency requirement on the procedures of LQC. As reported in [22], the self consistency indeed holds and we elaborate the proof in this paper.

The paper is organized as follows.

In section II, we recall the relevant details of the classical and the loop quantized description of the vacuum Bianchi IX model. We describe the kinematical set-up, the Hamiltonian constraint and the definition of inverse triad components including a class of quantization ambiguities.

In section III, we recall the continuum approximation leading to the Wheeler-DeWitt differential equation. We describe how the effective continuum Hamiltonian is obtained and how the most important non-perturbative corrections from the underlying quantum equations 
are incorporated. We also comment on the validity of the effective description.

In section IV, we display the effective Hamiltonian for the Bianchi IX model and specify the dynamics with respect to an internal time obtained by solving the effective Hamiltonian constraint.

In section $\mathrm{V}$, we analyze the potential term in the Hamiltonian for internal dynamics. This section is divided into four subsections. In subsection A, we describe the 'walls' (positive potential) of the potential. Unlike the classical one, this modified potential has a non-factorizable dependence on internal time. The walls therefore not only move but also

change their extent and height, eventually disappearing completely. Subsection B contains a demonstration of the stability of the Kasner solution when the effective potential is turned on. In subsection $\mathrm{C}$, we relate the Kasner solution in terms of the internal time to the more common description in terms of the scale factor evolution. In the last subsection, we specify the domain of validity of the effective description in quantitative terms.

In section VI, we extrapolate beyond the domain of validity mainly to contrast the usual oscillatory behavior under the influence of the classical potential. The (small volume) asymptotic analysis of this section uses qualitative arguments and proceeds in two main steps. In the first step, we rule out motion in the anisotropy plane being confined to a bounded region. In the second step we show that the asymptotic trajectory must approach a Kasner trajectory exponentially.

In section VII, we summarize our results. We also point out the insensitivity of the results to quantization ambiguities and discuss possible implications of the modified dynamics as to the structure of initial and final singularities.

\section{THE BIANCHI IX MODEL}

Bianchi models are a subclass of spatially homogeneous models such that the symmetry group contains a subgroup with simply transitive action on the spatial manifold. The simply transitive subgroups are classified in terms of three integers, $n^{I}$, parameterizing the structure constants as

$$
C_{J K}^{I}=\epsilon_{(I) J K} n^{I}
$$

Bianchi IX class of models have $n^{I}=1, I=1,2,3$. Clearly, $C_{J I}^{I}=0$ implying that Bianchi IX is a class A model admitting a canonical formulation. 
Given the subgroup acting simply transitively, one introduces the corresponding leftinvariant 1-forms $\omega^{I}=\omega_{a}^{I} \mathrm{~d} x^{a}$ and their dual, density weighted vector fields $X_{I}=X_{I}^{a} \partial_{a}$, on the spatial manifold $\Sigma$. The 1-forms satisfy the Maurer-Cartan equations,

$$
\mathrm{d} \omega^{I}=-\frac{1}{2} C_{J K}^{I} \omega^{J} \wedge \omega^{K}
$$

In the metric description, the metrics of Bianchi IX models have the form,

$$
d s^{2}=d t^{2}-\gamma_{I J}(t) \omega^{I} \omega^{J}
$$

while in the connection formulation, the geometry is described in terms of the invariant connections and densitized triads given by,

$$
A_{a}^{i}=\phi_{I}^{i}(t) \omega_{a}^{I}, \quad E_{i}^{a}=p_{i}^{I}(t) X_{I}^{a}
$$

with spatially constant and canonically conjugate $\phi_{I}^{i}, p_{i}^{I}$.

Note that while the symmetry group 'fixes' the invariant 1-forms only up to orthogonal transformations which preserve the structure constants of Bianchi IX, this freedom can be used only at one instant of time during an evolution. The $t$ dependence resides in the symmetric $\gamma_{I J}$ in the metric formulation and in $\phi_{I}^{i}, p_{i}^{I}$ in the connection formulation.

In the vacuum case, the Einstein equations $R_{0 a}=0$, imply that the matrix $\gamma_{I J}$ can be taken to be diagonal so that only three degrees of freedom remain in the metric formulation. In the presence of matter, the metric is in general non-diagonal. However, as argued by BKL, near the singularity, the non-diagonal terms do not affect either the nature of Kasner epochs (time intervals during which scales of spatial distances along three specific directions change as in the case of the Kasner solution), or the 'law of replacement of Kasner exponents'. Instead, these terms lead to rotation of the Kasner axes themselves during a change over from one Kasner epoch to another. Thus, to study the 'oscillatory behavior' implied by succession of Kasner epochs with changes in the exponents, it is sufficient to restrict to the sub-class of diagonal Bianchi IX, and anyway for the vacuum case this restriction is automatically implied. One now has only three degrees of freedom in the metric formulation.

In the connection formulation the situation is analogous. To begin with one has nine degrees of freedom, $\phi_{I}^{i}$ but with a three parameter gauge freedom of $\mathrm{SU}(2)$ rotations of the index $i$. A further freedom, depending upon the parameters $n^{I}$, would have been available if the left-invariant 1-forms were not thought of as a background structure in parameterizing 
the invariant connections. To get the same three degrees of freedom as in the diagonal metric formulation, one then restricts the $\phi_{I}^{i}$ to a "diagonal form", $\phi_{I}^{i}:=c_{(I)} \Lambda_{I}^{i}$ and correspondingly

$p_{i}^{I}:=p^{I} \Lambda_{i}^{(I)}$ where the $\mathrm{SO}(3)$-matrix $\Lambda$ includes gauge degrees of freedom (see [16] for details). Such a restriction specifies the diagonal models in the connection formulation. This is not a symmetry reduction in the same sense as restriction to isotropic models is. Nevertheless, these restricted models can be analyzed by following procedures similar to those in the context of symmetry reductions [23]. As in reductions to isotropic models, this leads to significant simplifications in such diagonalized models because the gauge parameters in $\Lambda$ and (almost; see below) gauge invariant parameters, $c_{I}$, are neatly separated [16].

\section{A. Classical Framework}

The basic variables for diagonal Bianchi Class A models are specified via

$$
A_{a}^{i}=c_{(I)} \Lambda_{I}^{i} \omega_{a}^{I}
$$

in terms of the 'gauge invariant' coefficients $c_{I}$ with the pure gauge degrees of freedom contained in the $S O(3)$-matrix $\Lambda$. The components $c_{I}$ are not completely gauge invariant but subject to residual discrete gauge transformations which change the sign of two of the three components simultaneously. A diagonal densitized triad has the form

$$
E_{i}^{a}=p^{(I)} \Lambda_{i}^{I} X_{I}^{a}
$$

where $X_{I}$ are left-invariant densitized vector fields dual to $\omega^{I}$. Being an $\operatorname{SO}(3)$-matrix, $\Lambda$ satisfies

$$
\Lambda_{I}^{i} \Lambda_{i}^{J}=\delta_{I}^{J}, \epsilon_{i j k} \Lambda_{I}^{i} \Lambda_{J}^{j} \Lambda_{K}^{k}=\epsilon_{I J K}
$$

The triad components $p^{I}$ are subject to the same residual gauge transformations as the connection components $c_{I}$ to which they are conjugate with basic Poisson bracket

$$
\left\{c_{I}, p^{J}\right\}=\gamma \kappa \delta_{I}^{J}
$$

where $\gamma$ is the Barbero-Immirzi parameter and $\kappa=8 \pi G$ the gravitational constant. We will use the value $\gamma=\frac{\log (2)}{\pi \sqrt{3}} \approx 0.13$ fixed by the black hole entropy calculations [24, 25].

A diagonal co-triad has the form $e_{a}^{i}=a_{(I)} \Lambda_{I}^{i} \omega_{a}^{I}$ with

$$
p^{1}=\left|a_{2} a_{3}\right| \operatorname{sgn}\left(a_{1}\right) \quad, \quad p^{2}=\left|a_{1} a_{3}\right| \operatorname{sgn}\left(a_{2}\right) \quad, \quad p^{3}=\left|a_{1} a_{2}\right| \operatorname{sgn}\left(a_{3}\right)
$$


Note that the components $p^{I}$ as well as $a_{I}$ can take negative values, but only the overall sign $\operatorname{sgn}\left(p^{1} p^{2} p^{3}\right)$ (i.e., the orientation) and the absolute values $\left|p^{I}\right|$ are gauge invariant. With this form of the co-triad, we obtain, in fact, a diagonal metric

$$
\mathrm{d} s^{2}=e_{I}^{i} e_{J}^{i} \omega^{I} \omega^{J}=\sum_{I} a_{I}^{2}\left(\omega^{I}\right)^{2}
$$

The extrinsic curvature and the spin connection components likewise have a diagonal form: $k_{a}^{i}:=K_{(I)} \Lambda_{I}^{i} \omega_{a}^{I}$ and $\Gamma_{a}^{i}:=\Gamma_{(I)} \Lambda_{I}^{i} \omega_{a}^{I}$ with $K_{I}=-\frac{1}{2} \dot{a}_{I}$ which appear in the relation $c_{I}=\Gamma_{I}-\gamma K_{I}$ defining the Ashtekar connection components $c_{I}$ in terms of the spin connection components $\Gamma_{I}$ and the extrinsic curvature. The spin connection components for Bianchi IX are given by [16],

$$
\begin{aligned}
\Gamma_{I} & =\frac{1}{2}\left(\frac{a_{J}}{a_{K}}+\frac{a_{K}}{a_{J}}-\frac{a_{I}^{2}}{a_{J} a_{K}}\right) \\
& =\frac{1}{2}\left(\frac{p^{K}}{p^{J}}+\frac{p^{J}}{p^{K}}-\frac{p^{J} p^{K}}{\left(p^{I}\right)^{2}}\right) \text { for }(I, J, K) \text { an even permutation of }(1,2,3) .
\end{aligned}
$$

Note that in contrast to the full theory, the spin connection is a covariant object within a homogeneous model since coordinate transformations have to respect the symmetry. Consequently, if non-zero, it cannot be made small by choosing appropriate local coordinates.

The Hamiltonian constraint [16] is given by,

$$
\begin{aligned}
H= & 2 \kappa^{-1}\left\{\left[\left(c_{2} \Gamma_{3}+c_{3} \Gamma_{2}-\Gamma_{2} \Gamma_{3}\right)\left(1+\gamma^{-2}\right)-c_{1}-\gamma^{-2} c_{2} c_{3}\right] a_{1}\right. \\
& +\left[\left(c_{1} \Gamma_{3}+c_{3} \Gamma_{1}-\Gamma_{1} \Gamma_{3}\right)\left(1+\gamma^{-2}\right)-c_{2}-\gamma^{-2} c_{1} c_{3}\right] a_{2} \\
& \left.+\left[\left(c_{1} \Gamma_{2}+c_{2} \Gamma_{1}-\Gamma_{1} \Gamma_{2}\right)\left(1+\gamma^{-2}\right)-c_{3}-\gamma^{-2} c_{1} c_{2}\right] a_{3}\right\} \\
= & 2 \kappa^{-1}\left[\left(\Gamma_{2} \Gamma_{3}-\Gamma_{1}\right) a_{1}+\left(\Gamma_{1} \Gamma_{3}-\Gamma_{2}\right) a_{2}+\left(\Gamma_{1} \Gamma_{2}-\Gamma_{3}\right) a_{3}\right. \\
& \left.-\frac{1}{4}\left(a_{1} \dot{a}_{2} \dot{a}_{3}+a_{2} \dot{a}_{1} \dot{a}_{3}+a_{3} \dot{a}_{1} \dot{a}_{2}\right)\right] .
\end{aligned}
$$

In order to derive the classical field equations (for which we consider only positive $a_{I}$ and $\left.p^{I}\right)$ it is advantageous to transform to new canonical variables

$$
\begin{array}{lll}
\pi_{I}:=2 K_{I} p^{(I)}=-\dot{a}_{I} a_{I}^{-1} a_{1} a_{2} a_{3}=-\left(\log a_{I}\right)^{\prime} & \text { and } \\
q^{I}:=\frac{1}{2} \log p^{I} & \text { such that } \quad & \left\{q^{I}, \pi_{J}\right\}=\kappa \delta_{I}^{J}
\end{array}
$$

where the prime denotes a derivative with respect to a new time coordinate $\tau$ related to $t$ by $\mathrm{d} t=a_{1} a_{2} a_{3} \mathrm{~d} \tau$ (corresponding to the lapse function $\left.N=a_{1} a_{2} a_{3}\right)$. With these new variables 
we have $\left\{\pi_{I}, p^{J}\right\}=-2 \kappa p^{(I)} \delta_{I}^{J}$ and

$$
\begin{aligned}
\kappa N H= & \kappa a_{1} a_{2} a_{3} H=2\left[p^{1} p^{2}\left(\Gamma_{1} \Gamma_{2}-\Gamma_{3}\right)+p^{1} p^{3}\left(\Gamma_{1} \Gamma_{3}-\Gamma_{2}\right)+p^{2} p^{3}\left(\Gamma_{2} \Gamma_{3}-\Gamma_{1}\right)\right. \\
& \left.-\frac{1}{4}\left(\pi_{1} \pi_{2}+\pi_{1} \pi_{3}+\pi_{2} \pi_{3}\right)\right] \\
= & \frac{1}{2}\left[\left(\frac{p^{2} p^{3}}{p^{1}}\right)^{2}+\left(\frac{p^{1} p^{3}}{p^{2}}\right)^{2}+\left(\frac{p^{1} p^{2}}{p^{3}}\right)^{2}\right] \\
& -\left(p^{3}\right)^{2}-\left(p^{2}\right)^{2}-\left(p^{1}\right)^{2}-\frac{1}{2}\left(\pi_{1} \pi_{2}+\pi_{1} \pi_{3}+\pi_{2} \pi_{3}\right) \\
= & \frac{1}{2}\left(a_{1}^{4}+a_{2}^{4}+a_{3}^{4}\right)-a_{1}^{2} a_{2}^{2}-a_{1}^{2} a_{3}^{2}-a_{2}^{2} a_{3}^{2}-\frac{1}{2}\left(\pi_{1} \pi_{2}+\pi_{1} \pi_{3}+\pi_{2} \pi_{3}\right) .
\end{aligned}
$$

Now one can easily derive the equations of motion,

$$
\left(\log a_{I}\right)^{\prime \prime}=-\pi_{I}^{\prime} \approx-\left\{\pi_{I}, a_{1} a_{2} a_{3} H\right\}
$$

which yield

$$
\begin{aligned}
& \frac{1}{2}\left(\log a_{1}\right)^{\prime \prime}=\left(a_{2}^{2}-a_{3}^{2}\right)^{2}-a_{1}^{4} \\
& \frac{1}{2}\left(\log a_{2}\right)^{\prime \prime}=\left(a_{1}^{2}-a_{3}^{2}\right)^{2}-a_{2}^{4} \\
& \frac{1}{2}\left(\log a_{3}\right)^{\prime \prime}=\left(a_{1}^{2}-a_{2}^{2}\right)^{2}-a_{3}^{4}
\end{aligned}
$$

or, using $\left(q^{1}\right)^{\prime \prime}=-\frac{1}{2}\left(\pi_{2}^{\prime}+\pi_{3}^{\prime}\right)$,

$$
\left(q^{I}\right)^{\prime \prime}=-4 a_{I}^{2} p^{I} \Gamma_{I}
$$

For the Bianchi I model we would have the right hand sides of equation (14) being zero so that $\log a_{I}=\alpha_{I}\left(\tau-\tau_{0, I}\right)$. This implies the Kasner behavior $a_{I} \propto t^{\alpha_{I}}$ where $t=e^{\tau}$ and the constraint (eq.(11) with $\left.\Gamma_{I}=0\right)$ requires $0=\alpha_{1} \alpha_{2}+\alpha_{1} \alpha_{3}+\alpha_{2} \alpha_{3}=\frac{1}{2}\left(\left(\alpha_{1}+\alpha_{2}+\alpha_{3}\right)^{2}-\right.$ $\left.\alpha_{1}^{2}-\alpha_{2}^{2}-\alpha_{3}^{2}\right)$. The coefficients $\alpha_{I}$ can be rescaled by choosing a different $t(\tau)$ which can be fixed by requiring the conventional parameterization $\alpha_{1}+\alpha_{2}+\alpha_{3}=1=\alpha_{1}^{2}+\alpha_{2}^{2}+\alpha_{3}^{2}$. As usual, these equations can be solved only if one coefficient, say $\alpha_{1}$, is negative and the other two are positive. Correspondingly, one direction, the first, contracts whereas the other two expand toward larger time. When we approach the classical singularity at $t=0$, space shrinks only in two directions while the third one expands unboundedly. The total volume however continues to approach zero according to $a_{1} a_{2} a_{3} \propto t^{\alpha_{1}+\alpha_{2}+\alpha_{3}}=t$. In [16] it has been shown that the Kasner singularity disappears when the model is quantized along the lines of loop quantum cosmology. 
For Bianchi IX the evolution of the three triad components can be described as motion in a non-trivial potential given by

$$
W\left(p^{1}, p^{2}, p^{3}\right)=2\left\{p^{1} p^{2}\left(\Gamma_{1} \Gamma_{2}-\Gamma_{3}\right)+p^{1} p^{3}\left(\Gamma_{1} \Gamma_{3}-\Gamma_{2}\right)+p^{2} p^{3}\left(\Gamma_{2} \Gamma_{3}-\Gamma_{1}\right)\right\}
$$

which has infinite walls at small $p^{I}$ owing to the divergence of the spin connection components. The evolution can then be described approximately as a succession of Kasner epochs with intermediate reflections at the potential [26]. For the Bianchi IX model the reflections never stop and the classical evolution is believed to be chaotic [27].

\section{B. Loop Quantization}

Diagonal homogeneous loop quantum cosmology [16] is first formulated in the connection representation where an orthonormal basis is given by the $\hat{p}^{I}$-eigenstates

$$
\left|m_{1}, m_{2}, m_{3}\right\rangle:=\left|m_{1}\right\rangle \otimes\left|m_{2}\right\rangle \otimes\left|m_{3}\right\rangle
$$

with

$$
\langle c \mid m\rangle=\frac{\exp \left(\frac{1}{2} i m c\right)}{\sqrt{2} \sin \left(\frac{1}{2} c\right)} .
$$

Here we are already restricting ourselves to a separable subspace of the kinematical Hilbert space, which is non-separable [28]. All the information about the classical singularity is already contained in wave functions restricted to this subspace such that it suffices for our purposes.

The eigenvalues of the triad operators can be read off from

$$
\hat{p}^{I}\left|m_{1}, m_{2}, m_{3}\right\rangle=\frac{1}{2} \gamma \ell_{\mathrm{P}}^{2} m_{I}\left|m_{1}, m_{2}, m_{3}\right\rangle
$$

Using the basic operators $\hat{p}^{I}$ one can define the volume operator $\hat{V}=\sqrt{\left|\hat{p}^{1} \hat{p}^{2} \hat{p}^{3}\right|}$ which will be used later. Its eigenstates are also $\left|m_{1}, m_{2}, m_{3}\right\rangle$ with eigenvalues

$$
V\left(m_{1}, m_{2}, m_{3}\right)=\left(\frac{1}{2} \gamma \ell_{\mathrm{P}}^{2}\right)^{\frac{3}{2}} \sqrt{\left|m_{1} m_{2} m_{3}\right|} .
$$

A kinematical state $|s\rangle$ is described in the triad representation by coefficients $s_{m_{1}, m_{2}, m_{3}}$ defined via

$$
|s\rangle=\sum_{m_{1}, m_{2}, m_{3}} s_{m_{1}, m_{2}, m_{3}}\left|m_{1}, m_{2}, m_{3}\right\rangle
$$


For a state to be gauge invariant under the residual gauge transformations changing the sign of two $p$-components simultaneously, the coefficients $s_{m_{1}, m_{2}, m_{3}}$ have to satisfy

$$
s_{m_{1}, m_{2}, m_{3}}=s_{-m_{1},-m_{2}, m_{3}}=s_{m_{1},-m_{2},-m_{3}}=s_{-m_{1}, m_{2},-m_{3}} .
$$

These states are left invariant by the gauge invariant triad operators $\left|\hat{p}^{I}\right|$ and the orientation operator $\operatorname{sgn}\left(\hat{p}^{1} \hat{p}^{2} \hat{p}^{3}\right)$. In calculations it is often easier to work with non-gauge invariant states in intermediate steps and project to gauge invariant ones in the end.

Together with the basic derivative operators $\hat{p}^{I}$ we need multiplication operators which usually arise from (point) holonomies $h_{I}=\exp \left(c_{(I)} \Lambda_{I}^{i} \tau_{i}\right)=\cos \left(\frac{1}{2} c_{I}\right)+2 \Lambda_{I}^{i} \tau_{i} \sin \left(\frac{1}{2} c_{I}\right)$ with action

$$
\begin{aligned}
& \cos \left(\frac{1}{2} c_{1}\right)\left|m_{1}, m_{2}, m_{3}\right\rangle=\frac{1}{2}\left(\left|m_{1}+1, m_{2}, m_{3}\right\rangle+\left|m_{1}-1, m_{2}, m_{3}\right\rangle\right) \\
& \sin \left(\frac{1}{2} c_{1}\right)\left|m_{1}, m_{2}, m_{3}\right\rangle=-\frac{1}{2} i\left(\left|m_{1}+1, m_{2}, m_{3}\right\rangle-\left|m_{1}-1, m_{2}, m_{3}\right\rangle\right)
\end{aligned}
$$

and correspondingly for $c_{2}$ and $c_{3}$.

\section{Inverse triad operators}

From the basic operators we can build more complicated ones. We will later need a quantization of the spin connection which is a composite operator containing several triad operators. In particular, it also contains inverse powers of triad components which classically diverge at the singularity. Since the triad operators have a discrete spectrum containing zero, they do not have an inverse. However, general methods of quantum geometry and loop quantum cosmology [15, 29] imply that there exist well-defined operators quantizing inverse triad components. To obtain these operators one makes use of a classical reformulation, e.g.

$$
\left|p^{1}\right|^{-1}=(l \gamma \kappa)^{\frac{1}{l-1}}\left(\left\{c_{1},\left|p^{1}\right|^{l}\right\}\right)^{\frac{1}{1-l}}=(l \gamma \kappa)^{\frac{1}{l-1}}\left(\frac{\operatorname{tr}_{j} \Lambda_{1}^{i} \tau_{i} h_{1}\left\{h_{1}^{-1},\left|p^{1}\right|^{l}\right\}}{\frac{1}{3} j(j+1)(2 j+1)}\right)^{\frac{1}{1-l}}
$$

which can then be quantized to

$$
\widehat{\left|p^{1}\right|_{j, l}^{-1}}=\mathrm{i}^{\frac{1}{l-1}}\left[\gamma \ell_{\mathrm{P}}^{2} l j(j+1)(2 j+1) / 3\right]^{\frac{1}{l-1}}\left(\operatorname{tr}_{j} \Lambda_{1}^{i} \tau_{i} h_{1}\left[h_{1}^{-1},\left|\hat{p}^{1}\right|^{l}\right]\right)^{\frac{1}{1-l}} \text {. }
$$

Here we have indicated that there are quantization ambiguities [30, 31] when one quantizes composite operators. The two most relevant for this paper are indicated by the subscript $(j, l)$. The half integer $j$ appearing as a subscript of the trace corresponds to the choice 
of representation while writing holonomies as multiplicative operators. The real valued $l \in(0,1)$, labels the various classically equivalent ways of writing $|p|^{-1}$ as a Poisson bracket of positive powers of $|p|$.

This operator acts as

$$
\begin{aligned}
\widehat{\left|p^{I}\right|_{j, l}^{-1}}\left|m_{1}, m_{2}, m_{3}\right\rangle & :=(2 l)^{\frac{1}{l-1}}\left(\frac{1}{2} \gamma \ell_{p}^{2}\right)^{-1} \mathcal{N}_{j}^{\frac{1}{1-l}} f_{j, l}\left(m_{I}\right)\left|m_{1}, m_{2}, m_{3}\right\rangle \quad \text { where } \\
\mathcal{N}_{j} & :=\frac{j(j+1)(2 j+1)}{3} \text { and } \\
f_{j, l}\left(m_{I}\right) & :=\left\{\sum_{k=-j}^{j} k\left(\left|m_{I}+2 k\right|\right)^{l}\right\}^{\frac{1}{1-l}} .
\end{aligned}
$$

The discrete values $f_{j, l}(m)$ decrease toward lower values for $m<2 j$ [30]. Thus, one can see that the classical divergence of the inverse of $\left|p^{I}\right|$ at vanishing $p^{I}$ is explicitly absent in the quantized operator. This will be seen to have further consequences for the approach to the classical singularity. Furthermore, the state $\left|m_{1}, m_{2}, m_{3}\right\rangle$ with $m_{I}=0$ (on which the classical inverse triad would diverge) is actually annihilated by $\widehat{\left|p^{I}\right|_{j, l}^{-1}}$ due to $f_{j, l}(0)=0$ independently of the ambiguities $j$ and $l$ in their allowed ranges. ${ }^{1}$ This allows us to define the inverse triad operator (not just its absolute value) by $\widehat{\left(p^{I}\right)_{j, l}^{-1}}:=\operatorname{sgn}\left(\hat{p}^{I}\right) \widehat{\left|p^{I}\right|_{j, l}^{-1}}$ without ambiguity in defining the sign of zero. These inverse triad operators will be used later to find well-defined operators quantizing the spin connection. Their eigenvalues are

$$
\widehat{\left(p^{I}\right)_{j, l}^{-1}}\left|m_{1}, m_{2}, m_{3}\right\rangle=(2 l)^{\frac{1}{l-1}}\left(\frac{1}{2} \gamma \ell_{p}^{2}\right)^{-1} \mathcal{N}_{j}^{\frac{1}{1-l}} \operatorname{sgn}\left(m_{I}\right) f_{j, l}\left(m_{I}\right)\left|m_{1}, m_{2}, m_{3}\right\rangle .
$$

It turns out to be convenient to define another function $F_{j, l}(m)$ as:

$$
F_{j, l}\left(m_{I}\right):=2 j\left(2 l \mathcal{N}_{j}\right)^{\frac{1}{l-1}} f_{j, l}\left(m_{I}\right)
$$

so that the eigenvalues are obtained as,

$$
\widehat{\left(p^{I}\right)_{j, l}^{-1}}\left|m_{1}, m_{2}, m_{3}\right\rangle=\left(\frac{1}{2} \gamma \ell_{\mathrm{p}}^{2}\right)^{-1}(2 j)^{-1} \operatorname{sgn}\left(m_{I}\right) F_{j, l}\left(m_{I}\right)\left|m_{1}, m_{2}, m_{3}\right\rangle .
$$

Then, for large $j$, using also $\mathcal{N}_{j} \sim \frac{2 j^{3}}{3}, F_{j, l}\left(m_{I}\right)=F_{l}\left(\mu_{I}\right)$ actually turns out to be a function of $\mu_{I}:=\frac{m_{I}}{2 j}$ and with $n o$ dependence on $j$. Explicitly [30, 31],

$$
F_{l}(q):=(2 l(l+1)(l+2) / 3)^{\frac{1}{l-1}}\left[(l+1)\left\{(q+1)^{l+2}-|q-1|^{l+2}\right\}\right.
$$

\footnotetext{
1 The usual case with eigenvalues $m_{I}^{-1}$, which corresponds to a discrete version of the Wheeler-DeWitt operator, could be obtained formally from (26) with the values $l=2, j=1 / 2$ which are not allowed here, and which would not result in an operator annihilating the states corresponding to the classical singularity.
} 


$$
\begin{aligned}
\left.-(l+2) q\left\{(q+1)^{l+1}-\operatorname{sgn}(q-1)|q-1|^{l+1}\right\}\right]^{\frac{1}{1-l}} \\
\longrightarrow\left[\frac{3 q}{l+1}\right]^{\frac{1}{1-l}} \quad(q \gg 1) \\
f_{j, l}\left(m_{I}\right) \approx\left(2^{l+1} l / 3\right)^{\frac{1}{1-l}} j^{\frac{2+l}{1-l}} F_{l}\left(\mu_{I}:=\frac{m_{I}}{2 j}\right)+o\left(j^{\frac{1+2 l}{1-l}}\right) .
\end{aligned}
$$

There is no suffix $j$ on $F_{l}$ since it is manifestly independent of $j$. The limiting forms show that for $\mu_{I} \gg 1$ one has the classical behavior for the inverse triad components and the quantum modifications are manifest for $\mu_{I}<1$ (or for $m_{I}<2 j$ ).

The inverse triad operators are used in defining the spin connection operators given in eq.(17) (specialized to Bianchi IX) and the corresponding potential (15). These are diagonal in the triad representation with eigenvalues given by,

$$
\begin{aligned}
\Gamma_{I}(\vec{\mu}) & =\frac{1}{2}\left\{\mu_{K} \operatorname{sgn}\left(\mu_{J}\right) F_{l}\left(\mu_{J}\right)+\mu_{J} \operatorname{sgn}\left(\mu_{K}\right) F_{l}\left(\mu_{K}\right)-\mu_{J} \mu_{K} F_{l}^{2}\left(\mu_{I}\right)\right\} \\
\frac{W_{j, l}(\vec{\mu})}{\left(\frac{1}{2} \gamma \ell_{\mathrm{p}}^{2}\right)^{2}} & =8 j^{2}\left[\mu_{I} \mu_{J}\left(\Gamma_{I} \Gamma_{J}-\Gamma_{K}\right)+\text { cyclic }\right]
\end{aligned}
$$

Note that the $\Gamma_{I}$, as functions of $\mu_{I}$, are both dimensionless and independent of $j$, while the potential explicitly scales as $j^{2}$. For $\mu_{I} \gg 1$, the $\Gamma_{I}$ become homogeneous functions of degree zero of their arguments, an observation which will be useful while discussing the effective Hamiltonian. To avoid proliferation of labels, we have suppressed the ambiguity label $l$ on the spin connection but have displayed both $j, l$ on the effective potential.

\section{The Hamiltonian constraint}

The quantization of the Hamiltonian constraint is somewhat more complicated in the presence of a non-zero spin connection. The details can be found in [19]. Here we summarize only the salient points.

The key difference between the full theory and the homogeneous models with non-zero spatial curvature is that the spin connection is a tensor due to the restriction on the coordinate transformations preserving the homogeneity. Consequently, it cannot be made to vanish unlike in the full theory. There is thus no guidance from the full theory regarding the incorporation of the spin connection. This loss of guidance is compensated by invoking two criteria of admissibility of quantization of the Hamiltonian[21]. 
Recall that the underlying discrete nature of loop quantum gravity implies a difference equation form for imposition of the Hamiltonian constraint. The order of this equation determines the number of independent solutions. Among these, there must be those which are slowly varying over the discreteness scale which should correspond to the familiar continuum, classical geometry. In essence, the two criteria of admissibility stipulate that the difference equation implied by a quantization of the Hamiltonian, must admit a continuum approximation which in addition must be (locally) stable in a certain well defined sense. In 19], a quantization of the Hamiltonian constraint for all diagonal homogeneous models was proposed and shown to satisfy the admissibility criteria.

The quantized Hamiltonian operator is given explicitly as:

$$
\begin{aligned}
\hat{H}=4 i\left(\gamma \ell_{p}^{2} \kappa\right)^{-1} \sum_{I J K} \epsilon^{I J K} \operatorname{tr}[ & \left\{\gamma^{-2} \hat{h}_{I}(A, \Gamma) \hat{h}_{J}(A, \Gamma)\left(\hat{h}_{I}(A, \Gamma)\right)^{-1}\left(\hat{h}_{J}(A, \Gamma)\right)^{-1}\right. \\
& \left.\left.-2\left(\hat{\Gamma}_{I} \hat{\Gamma}_{J}-n^{L} \hat{\Gamma}_{L}\right) \Lambda_{I} \Lambda_{J}\right\}\left\{h_{K}(A)\left[h_{K}^{-1}(A), \hat{V}\right]\right\}\right]
\end{aligned}
$$

where

$$
\begin{aligned}
\hat{h}_{I}(A, \Gamma) & :=e^{c_{(I)} \Lambda_{I}^{i} \tau_{i}} e^{-\hat{\Gamma}_{(I)} \Lambda_{I}^{i} \tau_{i}} \\
\hat{\Gamma}_{I} & :=\frac{1}{2}\left[\hat{p}^{J} n^{K} \widehat{\left(p^{K}\right)^{-1}}+\hat{p}^{K} n^{J} \widehat{\left(p^{J}\right)^{-1}}-\hat{p}^{J} \hat{p}^{K} n^{I}\left(\widehat{\left(p^{I}\right)^{-1}}\right)^{2}\right]
\end{aligned}
$$

and the $\widehat{\left(p^{I}\right)^{-1}}$ are defined in equation (27). Note that $\hat{\Gamma}_{I}$ operators are diagonal in the triad basis with eigenvalues denoted by $\Gamma_{I}\left(m_{1}, m_{2}, m_{3}\right)$.

In the presence of non-zero spin connection it is convenient to define a new set of basis vectors:

$$
\left|m_{1}, \widetilde{m_{2},} m_{3}\right\rangle:=e^{-\frac{1}{2} i \sum_{I} m_{I} \Gamma_{I}\left(m_{1}, m_{2}, m_{3}\right)}\left|m_{1}, m_{2}, m_{3}\right\rangle
$$

Denoting $|s\rangle=\tilde{s}_{m_{1}, m_{2}, m_{3}}\left|\widetilde{m_{1}, m_{2}, m_{3}}\right\rangle, \hat{H}|s\rangle=0$, leads to a partial difference equation. This partial difference equation is quite complicated and is detailed in [19]. For the purposes of this paper we only need to look at the continuum approximation.

\section{EFFECTIVE CLASSICAL FRAMEWORK}

The effective classical Hamiltonian can be derived from the underlying quantum equation in two steps. First one derives a continuum approximation and in the second step derives 
an effective classical Hamiltonian by considering a WKB ansatz for the continuum wave function.

First, the fundamental difference equation is specialized for a slowly varying discrete solution in the continuum regime defined by $m_{I}:=p^{I} /\left(\frac{1}{2} \gamma \ell_{\mathrm{P}}^{2}\right) \gg 1$. The leading terms are then independent of the Barbero-Immirzi parameter $\gamma$ and lead to the usual Wheeler-DeWitt equation for an interpolating continuum wave function [21].

Explicitly, for a slowly varying discrete wave function, $\tilde{s}_{m_{1}, m_{2}, m_{3}}:=\tilde{S}\left(p^{I}\left(m_{I}\right)\right), p^{I}\left(m_{I}\right):=$ $\frac{1}{2} \gamma \ell_{\mathrm{P}}^{2} m_{I}$, the interpolating continuous wave function $\tilde{S}$ satisfies the Wheeler-DeWitt equation [19], ${ }^{2}$

$$
\begin{gathered}
{\left[2 \ell_{p}^{4} p^{1} p^{2} \frac{\partial^{2} \sqrt{\left|p^{1} p^{2} p^{3}\right|} \tilde{S}\left(p^{1}, p^{2}, p^{3}\right)}{\partial p^{1} \partial p^{2}}+\operatorname{cyclic}\right]+W_{j, l}\left(p^{1}, p^{2}, p^{3}\right) \sqrt{\left|p^{1} p^{2} p^{3}\right|} \tilde{S}\left(p^{1}, p^{2}, p^{3}\right)} \\
=-\kappa\left|p^{1} p^{2} p^{3}\right|^{\frac{3}{2}} \hat{\rho}^{\text {matter }}\left(p^{1}, p^{2}, p^{3}\right) \tilde{S}\left(p^{1}, p^{2}, p^{3}\right)
\end{gathered}
$$

Note that in the above equation, the $p^{I}$ dependence is obtained by replacing $m_{I} /(2 j)=$ $\mu_{I}=p^{I} /\left(j \gamma \ell_{\mathrm{P}}^{2}\right)$. This will be important in the context of the potential and the matter terms (see below).

In the second step, one takes the slowly varying wave function $\tilde{T}:=\sqrt{\left|p^{1} p^{2} p^{3}\right|} \tilde{S}$ to be of the WKB form $\tilde{T} \sim e^{\mathrm{i} A / \hbar}$. Then the real part of the equation, to the leading order, $\hbar^{0}$, gives a partial differential equation for the phase $A$, with the phase appearing only through its first derivatives with respect to $p^{I}$. Eq. (10) suggests the identification $p^{I} \frac{\partial A}{\partial p^{I}}:=\frac{1}{2 \kappa} \pi_{I}$. Replacing the derivatives of the phase in terms of $\pi_{I}$ in the partial differential equation converts it into an expression which has exactly the same form as the classical Hamiltonian (11), except for the potential carrying the labels $j, l$. In short, we obtain a Hamilton-Jacobi equation for the phase. Classical actions (corresponding to the Hamiltonian) along dynamical trajectories are well known to solve the Hamilton-Jacobi equation. Thus constructing slowly varying solutions of the Wheeler-DeWitt equation naturally leads to analyzing the trajectories determined by the classical Hamiltonian derived in the above manner.

The classicality of the Hamiltonian presumes that in (39) the potential and the matter term are of order $\hbar^{0}$. This feature however depends on the values of $p^{I} /\left(j \gamma \ell_{\mathrm{P}}^{2}\right)$, because the ambiguity labels $j, l$ control the $p^{I}$ dependence of the potential. For instance, taking the

\footnotetext{
${ }^{2}$ In the equation (65) of this reference, the coefficient of the first terms should be 1 instead of $\frac{1}{4}$
} 
value of $j$ to be large, allows us to divide the $m_{I} \gg 1$ continuum region into (i) $j \gg m_{I} \gg 1$ and (ii) $m_{I} \gg j \gg 1$ sub-regions. Since $F_{l}$ is a function of $m /(2 j)$, the two sub-regions are more conveniently demarcated as $p^{I} \ll j \gamma \ell_{\mathrm{P}}^{2}$ and $p^{I} \gg j \gamma \ell_{\mathrm{P}}^{2}$ respectively.

In region (ii) the eigenvalues of the inverse triad operators correspond to the classical values (inverses of triad eigenvalues) and consequently the spin connection acquires no dependence on $\gamma \ell_{\mathrm{P}}^{2}$ when $\mu_{I}$ are expressed in terms of the $p^{I}$. The potential then reduces to the exact classical expressions without any dependence on $\gamma \ell_{\mathrm{P}}^{2}$.

By contrast, in region (i) the $F_{l}\left(\mu_{I}\right)$ have a power law dependence with positive power. This makes the spin connection have a positive degree dependence on its arguments and introduces inverse powers of $\gamma \ell_{\mathrm{P}}^{2}$ in the potential (and matter) term when $\mu_{I}$ are replaced by $p^{I} /\left(j \gamma \ell_{\mathrm{P}}^{2}\right)$. These terms clearly reflect non-perturbative quantum effects in region (i).

Thus the argument that the effective Hamiltonian derived via the WKB ansatz is classical $\left(\hbar^{0}\right)$ is strictly valid only in region (ii). Noting that in region (i), the inverse triads go to zero as $p^{I} \rightarrow 0$, we will assume the validity of the effective Hamiltonian in region (i) even though the potential has inverse powers of $\gamma \ell_{\mathrm{P}}^{2}$. In this manner, in an approximate sense, we incorporate non-perturbative modifications of the potential in an otherwise classical description.

The validity of the classical description is controlled by the validity of the WKB approximation while how much of quantum modifications can be seen in the classical framework is controlled by $j$. Similar remarks apply to the matter Hamiltonian terms since the matter density also typically gets quantum modifications from the inverse volume operator.

The classical Hamiltonian deduced from the WKB approximation in the above manner, including the extension to region (i), is what we will now refer to as effective Hamiltonian. Notice that imposition of the underlying quantum Hamiltonian constraint has automatically implied that the effective Hamiltonian must be zero which we interpret as the (modified) classical Hamiltonian constraint.

To study the dynamical implications of the effective Hamiltonian constraint, recall first that in general relativity we can have two distinct notions of dynamics or time evolution (see, e.g., [32]). One is evolution with respect to an external time which is a suitable coordinate in the space-time description of the geometry and the other is evolution with respect to an internal time which is one of the degrees of freedom treated as a 'clock'. The latter is obtained by solving the Hamiltonian constraint for one suitably chosen momentum variable, 
say some $\pi_{0}$, which becomes a function of the remaining phase space coordinates and the conjugate coordinate $q^{0}$ identified as the internal time. The evolution of the remaining degrees of freedom with respect to $q^{0}$ is specified by the new Hamiltonian, $\mathcal{H}:=-\pi_{0}$, which is in general time $\left(q^{0}\right)$ dependent. Given a solution of the internal dynamics, one can systematically construct a corresponding solution of an external dynamics. What a quantum Hamiltonian constraint does is to provide a modified classical Hamiltonian constraint - our effective Hamiltonian - via the continuum approximation as outlined above. This results in the modification of the trajectories of the internal dynamics and correspondingly of the trajectories of the external dynamics. In the context of homogeneous models, the internal dynamics turns out to be time dependent but an un-constrained system.

In the subsequent sections, we will analyze the internal dynamics determined from the modified Hamiltonian constraint.

\section{INTERNAL TIME EVOLUTION}

We begin with the effective Hamiltonian (without matter) obtained from the continuum approximation,

$$
\kappa N H_{j, l}^{\mathrm{eff}}\left(\pi_{I}, q^{I}\right)=-\frac{1}{2}\left(\pi_{1} \pi_{2}+\pi_{2} \pi_{3}+\pi_{3} \pi_{1}\right)+W_{j, l}\left(e^{2 q^{1}}, e^{2 q^{2}}, e^{2 q^{3}}\right) \quad, \quad\left\{q^{I}, \pi_{J}\right\}=\kappa \delta_{J}^{I} .
$$

Here, we are using dimensionless variables (or equivalently set the unit of length such that $\gamma \ell_{\mathrm{P}}^{2}=2$ ) and have put $m_{I}:=e^{2 q^{I}}>0$. The potential is given by eq. (34) with the spin connections given in eq.(33).

First we diagonalise the kinetic term in the Hamiltonian.

$$
\begin{gathered}
-\frac{1}{2}\left(\pi_{1} \pi_{2}+\pi_{2} \pi_{3}+\pi_{3} \pi_{1}\right):=-\frac{1}{4} \pi^{T} A \pi \quad:=-\frac{1}{4} \pi^{T} \text { ODO } \pi \text { where, } \\
A:=\left(\begin{array}{lll}
0 & 1 & 1 \\
1 & 0 & 1 \\
1 & 1 & 0
\end{array}\right)=\left(\begin{array}{ccc}
\frac{2}{\sqrt{6}} & 0 & \frac{1}{\sqrt{3}} \\
\frac{-1}{\sqrt{6}} & \frac{1}{\sqrt{2}} & \frac{1}{\sqrt{3}} \\
\frac{-1}{\sqrt{6}} & -\frac{1}{\sqrt{2}} & \frac{1}{\sqrt{3}}
\end{array}\right)\left(\begin{array}{ccc}
-1 & 0 & 0 \\
0 & -1 & 0 \\
0 & 0 & 2
\end{array}\right)\left(\begin{array}{ccc}
\frac{2}{\sqrt{6}} & -\frac{1}{\sqrt{6}} & -\frac{1}{\sqrt{6}} \\
0 & \frac{1}{\sqrt{2}} & -\frac{1}{\sqrt{2}} \\
\frac{1}{\sqrt{3}} & \frac{1}{\sqrt{3}} & \frac{1}{\sqrt{3}}
\end{array}\right)
\end{gathered}
$$

Defining $\pi^{\prime}:=O^{T} \pi, q^{\prime}:=O^{T} q$ we get the kinetic term as $-\frac{1}{2} \pi_{0}^{2}+\frac{1}{4}\left(\pi_{+}^{2}+\pi_{-}^{2}\right)$ and the potential is obtained as a function of $q^{\prime}=:\left(q^{+}, q^{-}, q^{0}\right)$ by substituting $q=O q^{\prime}$. Explicitly,

$$
q^{1}=\frac{2}{\sqrt{6}} q^{+}+\frac{1}{\sqrt{3}} q^{0} \quad \quad \mu_{1}=\frac{1}{2 j} e^{\frac{2}{\sqrt{3}} q^{0}} e^{\frac{4}{\sqrt{6}} q^{+}}
$$




$$
\begin{aligned}
q^{2} & =-\frac{1}{\sqrt{6}} q^{+}+\frac{1}{\sqrt{2}} q^{-}+\frac{1}{\sqrt{3}} q^{0} & \mu_{2} & =\frac{1}{2 j} e^{\frac{2}{\sqrt{3}} q^{0}} e^{-\frac{2}{\sqrt{6}} q^{+}} e^{\sqrt{2} q^{-}} \\
q^{3} & =-\frac{1}{\sqrt{6}} q^{+}-\frac{1}{\sqrt{2}} q^{-}+\frac{1}{\sqrt{3}} q^{0} & \mu_{3} & =\frac{1}{2 j} e^{\frac{2}{\sqrt{3}} q^{0}} e^{-\frac{2}{\sqrt{6}} q^{+}} e^{-\sqrt{2} q^{-}}
\end{aligned}
$$

Clearly, $q^{0}=\frac{1}{\sqrt{3}}\left(q^{1}+q^{2}+q^{3}\right)=\frac{1}{\sqrt{3}} \ln$ (volume). For an internal time description, this is a suitable variable. The internal time description is obtained by solving $H_{j, l}^{\text {eff }}=0$ for $\pi_{0}$ :

$$
-\pi_{0}=\mathcal{H}\left(q^{+}, q^{-}, q^{0}, \pi_{+}, \pi_{-}\right):=\left[\frac{1}{2}\left(\pi_{+}^{2}+\pi_{-}^{2}\right)+2 W_{j, l}\left(q^{+}, q^{-}, q^{0}\right)\right]^{\frac{1}{2}}=: h^{\frac{1}{2}}
$$

$\mathcal{H}$ is to be used as the new Hamiltonian for analyzing the behavior of $q^{ \pm}$as the volume shrinks to zero monotonically i.e. as $q^{0} \rightarrow-\infty$. Note that there are no longer constraints but the new Hamiltonian is explicitly 'time' dependent. We are also suppressing the labels $j, l$ on $\mathcal{H}$ and $h$.

The equations of motion then take the form,

$$
\begin{gathered}
\frac{\mathrm{d} q^{ \pm}}{\mathrm{d} q^{0}}=\frac{1}{2 \sqrt{h}} \pi_{ \pm}, \quad \frac{\mathrm{d} \pi_{ \pm}}{\mathrm{d} q^{0}}=-\frac{1}{\sqrt{h}} \frac{\partial W_{j, l}}{\partial q^{ \pm}} \\
\frac{\mathrm{d}^{2} q^{ \pm}}{\mathrm{d}\left(q^{0}\right)^{2}}=-\frac{1}{2 h} \frac{\partial W_{j, l}}{\partial q^{ \pm}}-\frac{1}{h} \frac{\partial W_{j, l}}{\partial q^{0}} \frac{\mathrm{d} q^{ \pm}}{\mathrm{d} q^{0}}
\end{gathered}
$$

Along a solution of the equation of motion, we can eliminate $h$ using $h=2 h\left(\left(\dot{q}^{+}\right)^{2}+\right.$ $\left.\left(\dot{q}^{-}\right)^{2}\right)+2 W_{j, l}$ or $h=2 W_{j, l} /\left(1-2\left(\left(\dot{q}^{+}\right)^{2}+\left(\dot{q}^{-}\right)^{2}\right)\right)$. This leads to

$$
\frac{\mathrm{d}^{2} q^{ \pm}}{\mathrm{d}\left(q^{0}\right)^{2}}=-\left\{1-2\left(\frac{\mathrm{d} q^{+}}{\mathrm{d} q^{0}}\right)^{2}-2\left(\frac{\mathrm{d} q^{-}}{\mathrm{d} q^{0}}\right)^{2}\right\}\left\{\frac{1}{4 W_{j, l}} \frac{\partial W_{j, l}}{\partial q^{ \pm}}+\frac{1}{2 W_{j, l}} \frac{\partial W_{j, l}}{\partial q^{0}} \frac{\mathrm{d} q^{ \pm}}{\mathrm{d} q^{0}}\right\}
$$

It will also turn out to be convenient to introduce polar coordinates in the $q^{ \pm}$anisotropy plane. This is easily done: Define $q^{+}:=R \cos (\theta), q^{-}:=R \sin (\theta)$. The conjugate momenta then are given by $\pi_{+}:=\pi_{R} \cos (\theta)-\pi_{\theta} \frac{\sin (\theta)}{R}, \pi_{-}:=\pi_{R} \sin (\theta)+\pi_{\theta} \frac{\cos (\theta)}{R}$. The squared Hamiltonian, $h$, and the equations of motion are given by

$$
\begin{aligned}
h\left(R, \theta, \pi_{R}, \pi_{\theta}, q^{0}\right) & =\frac{1}{2}\left(\pi_{R}^{2}+R^{-2} \pi_{\theta}^{2}\right)+2 W_{j, l}\left(p^{I}\left(q^{0}, R, \theta\right)\right), \\
\mathcal{H}\left(R, \theta, \pi_{R}, \pi_{\theta}, q^{0}\right) & =\sqrt{h\left(R, \theta, \pi_{R}, \pi_{\theta}, q^{0}\right)} .
\end{aligned}
$$

Denoting the $\mathrm{d} / \mathrm{d} q^{0}$ by an over dot, the Hamilton's equations of motion take the form,

$$
\begin{gathered}
\dot{\theta}=\frac{1}{2 \sqrt{h}} R^{-2} \pi_{\theta}, \dot{R}=\frac{1}{2 \sqrt{h}} \pi_{R} \\
\dot{\pi_{\theta}}=-\frac{1}{\sqrt{h}}\left(\frac{\partial W_{j, l}}{\partial \theta}\right), \dot{\pi_{R}}=-\frac{1}{2 \sqrt{h}}\left(-R^{-3} \pi_{\theta}^{2}+2 \frac{\partial W_{j, l}}{\partial R}\right) .
\end{gathered}
$$


The second order equations are obtained as,

$$
\begin{aligned}
\ddot{R}-R \dot{\theta}^{2} & =-h^{-1}\left(\frac{1}{2} \frac{\partial W_{j, l}}{\partial R}+\dot{R} \frac{\partial W_{j, l}}{\partial q^{0}}\right), \\
\ddot{\theta}+\frac{2}{R} \dot{R} \dot{\theta} & =-h^{-1}\left(\frac{1}{2} R^{-2} \frac{\partial W_{j, l}}{\partial \theta}+\dot{\theta} \frac{\partial W_{j, l}}{\partial q^{0}}\right) .
\end{aligned}
$$

Along the dynamical trajectories, we can eliminate the conjugate momenta from the Hamiltonian and obtain,

$$
h^{-1}=\frac{1-2\left(\dot{R}^{2}+R^{2} \dot{\theta}^{2}\right)}{2 W_{j, l}}>0 \quad \text { (strict inequality). }
$$

When the potential is zero, as in Bianchi I, the right hand sides of (52, 53) are zero. It is easy to see that the Kasner solution is obtained as $\theta=$ constant, $R\left(q^{0}\right)=-\frac{1}{\sqrt{2}} q^{0}+$ constant. When the potential is non-zero, the equations are quite complicated. To proceed with the qualitative analysis, we will study the effective potential in the next section.

\section{STUDY OF THE EFFECTIVE POTENTIAL}

The effective potential is more conveniently studied in terms of the polar variables. The following expressions are useful for our subsequent analysis.

$$
\begin{aligned}
q^{+} & :=R \cos \theta & q^{-} & :=R \sin \theta \\
Q\left(j, q^{0}\right) & :=\frac{e^{\frac{2}{\sqrt{3}} q^{0}}}{2 j}=\frac{(\text { volume })^{2 / 3}}{2 j} & & \\
\mu_{1} & =Q e^{\frac{4}{\sqrt{6}} R \cos \theta} & & =Q e^{+\frac{4}{\sqrt{6}} R \cos \theta} \\
\mu_{2} & =Q e^{-\frac{2}{\sqrt{6}} R \cos \theta(1-\sqrt{3} \tan \theta)} & & =Q e^{-\frac{4}{\sqrt{6}} R \cos \left(\theta+\frac{\pi}{3}\right)} \\
\mu_{3} & =Q e^{-\frac{2}{\sqrt{6}} R \cos \theta(1+\sqrt{3} \tan \theta)} & & =Q e^{-\frac{4}{\sqrt{6}} R \cos \left(\theta-\frac{\pi}{3}\right)}
\end{aligned}
$$

Clearly, $\mu_{1} \mu_{2} \mu_{3}=Q^{3}$ is independent of the $q^{ \pm}$.

Using the obvious abbreviation, $F_{I}:=F_{l}\left(\mu_{I}\right)$, (label $l$ on $F$ is suppressed) the potential is given explicitly by,

$$
\begin{aligned}
\frac{W_{j, l}(\vec{\mu})}{2 j^{2}}= & {\left[-2\left\{\mu_{1} \mu_{2}\left(\mu_{1} F_{2}+\mu_{2} F_{1}\right)+\mu_{2} \mu_{3}\left(\mu_{2} F_{3}+\mu_{3} F_{2}\right)+\mu_{3} \mu_{1}\left(\mu_{3} F_{1}+\mu_{1} F_{3}\right)\right\}+\right.} \\
& 3\left\{\left(\mu_{1} \mu_{2} F_{3}\right)^{2}+\left(\mu_{2} \mu_{3} F_{1}\right)^{2}+\left(\mu_{3} \mu_{1} F_{2}\right)^{2}\right\}+3 Q^{3}\left\{\mu_{1} F_{2} F_{3}+\mu_{2} F_{3} F_{1}+\mu_{3} F_{1} F_{2}\right\} \\
& -Q^{3}\left\{\mu_{1} \mu_{2} F_{3}\left(F_{1}^{2}+F_{2}^{2}\right)+\mu_{2} \mu_{3} F_{1}\left(F_{2}^{2}+F_{3}^{2}\right)+\mu_{3} \mu_{1} F_{2}\left(F_{3}^{2}+F_{1}^{2}\right)\right\} \\
& \left.-2 Q^{3}\left\{\mu_{1} \mu_{2} F_{3}^{3}+\mu_{2} \mu_{3} F_{1}^{3}+\mu_{3} \mu_{1} F_{2}^{3}\right\}+Q^{6}\left\{F_{1}^{2} F_{2}^{2}+F_{2}^{2} F_{3}^{2}+F_{3}^{2} F_{1}^{2}\right\}\right]
\end{aligned}
$$


We define the far regime, by $\mu_{I} \gg 1$ and the near regime by $0<\mu_{I} \ll 1$. Note that the regimes are delineated in terms of the $\mu_{I}$ and not in terms of the anisotropy variables $q^{ \pm}$. The regimes are realized for any bounded region in the anisotropy plane $\left(q^{+}-q^{-}\right.$plane $)$and sufficiently large volume $(Q \gg 1)$ and sufficiently small volume $(Q \ll 1)$ respectively. In these regimes, the potential simplifies to the forms,

$$
\begin{gathered}
W_{j, l}^{\text {near }}(\vec{\mu}) \approx-4 j^{2}\left(\frac{3}{l+1}\right)^{\frac{1}{1-l}}\left[\mu_{3}^{\frac{2-l}{1-l}}\left(\mu_{1}^{2}+\mu_{2}^{2}\right)+\mu_{1}^{\frac{2-l}{1-l}}\left(\mu_{2}^{2}+\mu_{3}^{2}\right)+\mu_{2}^{\frac{2-l}{1-l}}\left(\mu_{3}^{2}+\mu_{1}^{2}\right)\right] \\
=-2(2 j)^{-\frac{2-l}{1-l}}\left(\frac{3}{l+1}\right)^{\frac{1}{1-l}} e^{\frac{2}{\sqrt{3}} q^{0}\left(\frac{4-3 l}{1-l}\right)}\left[e^{-\frac{2}{\sqrt{6}} q^{+}\left(\frac{4-3 l}{1-l}\right)} \cosh \left(\frac{l}{1-l} \sqrt{2} q^{-}\right)\right. \\
+e^{\frac{2}{\sqrt{6}} q^{+}\left(\frac{2-3 l}{1-l}\right)} \cosh \left(\frac{2-l}{1-l} \sqrt{2} q^{-}\right) \\
\left.+e^{\frac{4}{\sqrt{6}} q^{+}\left(\frac{1}{1-l}\right)} \cosh \left(2 \sqrt{2} q^{-}\right)\right] \\
W_{j, l}^{\mathrm{far}}(\vec{\mu}) \approx 2 j^{2}\left[\left(\frac{\mu_{1} \mu_{2}}{\mu_{3}}\right)^{2}+\left(\frac{\mu_{2} \mu_{3}}{\mu_{1}}\right)^{2}+\left(\frac{\mu_{3} \mu_{1}}{\mu_{2}}\right)^{2}-2\left(\mu_{1}^{2}+\mu_{2}^{2}+\mu_{3}^{2}\right)\right] \\
\approx \frac{1}{2} e^{\frac{4}{\sqrt{3}} q^{0}\left[e^{-\frac{16}{\sqrt{6}} q^{+}}-4 e^{-\frac{4}{\sqrt{6}} q^{+}} \cosh \left(2 \sqrt{2} q^{-}\right)\right.} \\
\left.+2 e^{\frac{8}{\sqrt{6}} q^{+}}\left\{\cosh \left(4 \sqrt{2} q^{-}\right)-1\right\}\right]
\end{gathered}
$$

Comparison of expression (59) with Misner's expressions [26] suggests the identification: $q^{+} \rightarrow \frac{\sqrt{6}}{2} \beta_{+}, q^{-} \rightarrow \frac{\sqrt{6}}{2} \beta_{-}, q^{0} \rightarrow-\sqrt{3} \Omega$. We will continue to work with the $q$ 's.

Note that although in the two limiting cases the $q^{0}$ dependence factors out in the potential, this is not true in general. This is different from the classical potential which is just the far form of the effective potential.

The factorization property of the far and near potential allows to show that there can be no bounce in the corresponding regimes, i.e., once the volume decreases, it must keep on decreasing until it reaches zero [33]. This follows from the external time dynamics generated by the constraint $H=-\pi_{0}^{2}+\frac{1}{2}\left(\pi_{+}^{2}+\pi_{-}^{2}\right)+2 W=0$. For $q^{0}$ and its conjugate the Hamiltonian equations of motion are $\dot{q}^{0}=-2 \pi_{0}$ and $\dot{\pi}_{0}=-\partial H / \partial q^{0}=-2 \partial W / \partial q^{0}=-2 k W$ with positive constants $k$ in the near and far regime. This implies

$$
\frac{\mathrm{d}}{\mathrm{d} t}\left(\pi_{0} e^{-k q^{0} / 2}\right)=k e^{-k q^{0} / 2}\left(\pi_{0}^{2}-2 W\right)=k e^{-k q^{0} / 2}\left(\pi_{+}^{2}+\pi_{-}^{2}\right) / 2 \geq 0 .
$$

Thus, once the volume decreases, i.e. $\dot{q}^{0}<0$ and $\pi_{0}>0$, it cannot turn around since $\pi_{0} e^{-k q^{0} / 2}$ starts out positive and cannot decrease. This proof, however, relies on the factorization of 
the potential into a part depending exponentially on $q^{0}$ and a part depending only on the anisotropies. In regions where such a factorization is not possible, it is not clear if bounces or oscillations are ruled out. The modification of the potential could lead to a bounce at positive volume as observed in [34] for a closed isotropic model with scalar matter. If a similar bounce would occur in the effective Bianchi IX dynamics, the system would clearly not show the classical chaotic behavior. Thus, we can assume here that there is no bounce in order to investigate the potentially chaotic behavior.

Other asymptotic regions correspond to two of the $\mu_{I}$ 's large (small) and the third one small (large). These are realized for example, for a fixed and moderate $Q$ and large $R$. The behavior of $\mu_{I}$ 's is then controlled by the angles. These regions divide the anisotropy plane into angular sectors with $R$ large. In these regions, we could use the asymptotic forms of $F_{l}\left(\mu_{I}\right)$ 's and obtain the corresponding approximate forms of the potential. These forms are of course different from the near and far forms displayed in equations (58, 591). These are discussed in more details in section VI.

\section{A. Approximate wall picture}

One can see the qualitative behavior of the potential (classical or effective) by specializing to $q^{-}=0$ or $\theta=0$ and $\pi$. For this $q^{-}$, we get $\mu_{2}=\mu_{3}:=\mu$ and $\mu_{1}:=\nu=\frac{Q^{3}}{\mu^{2}}$. It follows that $\Gamma_{2}=\Gamma_{3}$ and the potential simplifies a bit. A wall, i.e. positive potential, is seen for $\mu \gg 1$ so that $F_{l}(\mu) \approx \mu^{-1}$ may be used but $\nu$ is not so small that $F_{l}(\nu) \approx 4 \nu^{2}$ can be used. Making this substitution for $F_{l}(\mu)$ one obtains,

$$
\begin{aligned}
\Gamma_{3}=\Gamma_{2} & \approx \frac{1}{2} \mu F_{l}(\nu) \quad, \quad \Gamma_{1} \approx 1-\frac{1}{2} \mu^{2} F_{l}^{2}(\nu) ; \\
W_{j, l}(\nu, \mu, \mu) & \approx 2 j^{2} \mu^{4} F_{l}^{2}(\nu)\left\{3-2 \nu F_{l}(\nu)\right\}-8 j^{2} \mu^{2} \\
& \approx 2 j^{2} \frac{Q^{6}}{\nu^{2}} F_{l}^{2}(\nu)\left\{3-2 \nu F_{l}(\nu)\right\}-8 j^{2} \frac{Q^{3}}{\nu}, \\
& \left.=: V_{l}(Q, \nu)-8 j^{2} \frac{Q^{3}}{\nu} \quad \text { (definition of } V_{l}(Q, \nu)\right) .
\end{aligned}
$$

We refer to $W_{j, l}(\nu, \mu, \mu)$ in eq.(60) as the wall potential and to $V_{l}(Q, \nu)$ defined in (61) as the approximate wall potential.

The last term in the wall potential can be neglected for $\nu \sim o(1)$ and $Q^{3} / \nu=\mu^{2} \gg 1$, since $F_{l}^{2}(\nu)\left(3-2 \nu F_{l}(\nu)\right)$ is of the order of 1 for this range of values. Neglecting this term and 
substituting $Q^{6}=(\text { volume })^{4} /(2 j)^{6}$ in the approximate wall potential, gives the expression quoted in [22].

For $\nu \gg 1$, one can replace $F_{l}(\nu)$ by $\nu^{-1}$ as well leading to $V_{l}(Q, \nu) \approx 2 j^{2} Q^{6} / \nu^{4} \approx$ $\frac{1}{2}$ (volume $)^{4 / 3} e^{-\frac{16}{\sqrt{6}} q^{+}}$matching with the leading term of eq.(59) for $q^{-}=0$ which is the classical wall potential.

In contrast to the classical wall, the wall of the effective potential has finite height. The location of its maximum along the $q^{+}$axis is defined by $\frac{\partial V_{l}}{\partial q^{+}}=0$. Its solution will define a function $q^{+}(Q)$. The motion of this position is obtained by solving $\frac{\mathrm{d}}{\mathrm{d} Q}\left(\frac{\partial V_{l}}{\partial \nu}\right)=0$. Recall $\nu=Q e^{\frac{4}{\sqrt{6}} q^{+}}=: Q e^{x}$ such that

$$
\begin{aligned}
& \frac{\partial V_{l}(Q, \nu(Q, x))}{\partial x}=0 \Longleftrightarrow \frac{\partial V_{l}}{\partial \nu}=0 \\
& \frac{\partial^{2} V_{l}}{\partial x^{2}}<0 \quad \Longleftrightarrow \quad \frac{\partial^{2} V_{l}}{\partial \nu^{2}}<0
\end{aligned}
$$

Thus, a (local) maximum of the approximate wall potential can be defined either in terms of derivatives with respect to $x=\frac{4}{\sqrt{6}} q^{+}$or with respect to $\nu$.

Notice that for the classical wall, there is no local extremum and therefore one has to track the motion of a fixed value of the potential. Thus, when the volume decreases, $q^{+}$ must also decrease, i.e. the wall moves away from the isotropy neighborhood (note that the wall is seen for $\mu \gg 1$ which implies $q^{+}<0$ ). By contrast, the approximate wall potential does have a local maximum at some $\nu_{0}$. Fixed $\nu$ implies that, with decreasing volume, the height of the approximate wall decreases and $q^{+}$increases. Thus the approximate wall moves towards the isotropy neighborhood with decreasing height. Eventually of course the wall potential must be used and the height actually crosses zero for a certain volume depending upon the values of $j, l$.

It is difficult to obtain a convenient approximation for a wall along an arbitrary direction and numerics must be used. Numerically, the full effective potential becomes zero everywhere, in the anisotropy plane, for $Q \approx 1.08511$ or for the dimensionless volume of about $(2.172 j)^{3 / 2}$ (for the choice $l=\frac{1}{2}$ ).

From these studies, it is apparent that the principal reason for the usual chaotic behavior disappears suggesting that the usual 'Kasner epochs' will continue without any replacements once the volume becomes comparable to the Planck volume. This in turn suggests that the Kasner solution should be stable under the effective potential. 


\section{B. Stability of Kasner solution}

This is most easily done in the Cartesian form (47). Substitute $q^{ \pm}\left(q^{0}\right)=K^{ \pm}\left(q^{0}\right)+\delta q^{ \pm}\left(q^{0}\right)$ where $K^{ \pm}:=-\frac{1}{\sqrt{2}} u^{ \pm} q^{0}, u^{+}:=\cos (\phi), u^{-}:=\sin (\phi)$. Linearization gives,

$$
\begin{aligned}
\ddot{\delta q}^{ \pm} & =-\left.\left\{-4 \dot{K}^{+} \dot{\delta q}^{+}-4 \dot{K}^{-} \dot{\delta q}^{-}\right\}\left\{\frac{1}{4 W_{j, l}} \frac{\partial W_{j, l}}{\partial q^{ \pm}}+\dot{q}^{ \pm} \frac{1}{2 W_{j, l}} \frac{\partial W_{j, l}}{\partial q^{0}}\right\}\right|_{\text {Kasner }} \\
& =-\left.2 \sqrt{2}\left\{\cos \phi \dot{\delta}^{+}+\sin \phi \dot{\delta} q^{-}\right\}\left\{\frac{1}{4 W_{j, l}} \frac{\partial W_{j, l}}{\partial q^{ \pm}}-\frac{u^{ \pm}}{2 \sqrt{2}} \frac{1}{W_{j, l}} \frac{\partial W_{j, l}}{\partial q^{0}}\right\}\right|_{\text {Kasner }} \\
& :=\kappa^{ \pm}\left(q^{0}, \phi\right)\left\{\cos \phi \dot{\delta q}+\sin \phi \dot{\delta q} \dot{q}^{-}\right\} \\
\frac{\mathrm{d}}{\mathrm{d} q^{0}}\left(\begin{array}{c}
\dot{\delta q}^{+} \\
\dot{\delta q}^{-}
\end{array}\right) & =\left(\begin{array}{cc}
\kappa^{+} \cos \phi & \kappa^{+} \sin \phi \\
\kappa^{-} \cos \phi & \kappa^{-} \sin \phi
\end{array}\right)\left(\begin{array}{c}
\dot{\delta q}^{+} \\
\dot{\delta q}
\end{array}\right)
\end{aligned}
$$

It is obvious now that the determinant of the matrix is zero and the only (possibly) non-zero eigenvalue is given by the trace: $\lambda:=\kappa^{+} \cos \phi+\kappa^{-} \sin \phi$.

The eigenvector corresponding to the zero eigenvalue is given by $\dot{\delta q} \dot{q}^{-}=-\cot \phi \delta \dot{q}^{+}$. The first braces in the middle equation of (63) then vanishes. This zero mode implies that the perturbed solution is also a Kasner solution. But we know that the dynamical equations do not admit Kasner solution since the potential is generally non-zero and $h$ is to be finite and positive and this solution of the perturbed equation must be rejected.

Thus the Kasner solution is stable if $\lambda>0$ (positive because we are considering the backward evolution, decreasing $q^{0}$ ). Up to here, no specific form of potential has been used.

To compute $\kappa^{ \pm}$we need to compute the potential on the Kasner solution. Substituting Kasner $K^{ \pm}\left(q^{0}\right)$ in the $\mu_{I}$ gives,

$$
\begin{aligned}
& 2 j \mu_{1}=e^{\frac{2}{\sqrt{3}} q^{0}(1-\cos \phi)} \\
& 2 j \mu_{2}=e^{\frac{2}{\sqrt{3}} q^{0}\left(1+\frac{1}{2} \cos \phi-\frac{\sqrt{3}}{2} \sin \phi\right)} \\
& 2 j \mu_{3}=e^{\frac{2}{\sqrt{3}} q^{0}\left(1+\frac{1}{2} \cos \phi+\frac{\sqrt{3}}{2} \sin \phi\right)}
\end{aligned}
$$

It is obvious now that all the exponents are non-negative over all $\phi$ implying that all $\mu_{I}$ will (generically) decrease as $q^{0}$ is decreased. Only for $\phi=120^{0}$ where $\mu_{2}=1 /(2 j), \phi=240^{0}$ when $\mu_{3}=1 /(2 j)$ and $\phi=0^{0}$ where $\mu_{1}=1 /(2 j)$, all exponents are positive. By taking $j>1 / 2$ we can ensure that all the $\mu_{I}$ are much less than 1 at some initial $q^{0}$. We can then use the near-form of the potential to compute the derivatives. 
Defining, generally, $2 j \mu_{I}=: e^{\nu_{I}\left(q^{0}, q^{ \pm}\right)}$one can obtain the derivatives of the potential as,

$$
\begin{aligned}
\delta W_{j, l} & =\sum_{I} \delta \nu_{I} \mu_{I} \frac{\partial W_{j, l}}{\partial \mu_{I}} \quad \Rightarrow \\
\frac{\partial W_{j, l}}{\partial q^{0}} & =\frac{2}{\sqrt{3}} \sum_{I} \mu_{I} \frac{\partial W_{j, l}}{\partial \mu_{I}} \\
\frac{\partial W_{j, l}}{\partial q^{+}} & =\frac{2}{\sqrt{6}}\left(2 \mu_{1} \frac{\partial}{\partial \mu_{1}}-\mu_{2} \frac{\partial}{\partial \mu_{2}}-\mu_{3} \frac{\partial}{\partial \mu_{3}}\right) W_{j, l} \\
\frac{\partial W_{j, l}}{\partial q^{-}} & =\sqrt{2}\left(\mu_{2} \frac{\partial}{\partial \mu_{2}}-\mu_{3} \frac{\partial}{\partial \mu_{3}}\right) W_{j, l} \\
W_{j, l}^{\text {near }} & =-4 j^{2}\left(\frac{3}{l+1}\right)^{\frac{1}{1-l}}\left[\mu_{1}^{L}\left(\mu_{2}^{2}+\mu_{3}^{2}\right)+\mu_{2}^{L}\left(\mu_{3}^{2}+\mu_{1}^{2}\right)+\mu_{3}^{L}\left(\mu_{1}^{2}+\mu_{2}^{2}\right)\right]
\end{aligned}
$$

where $L=\frac{2-l}{1-l}$. The logarithmic derivatives of the near potential are given by,

$$
\begin{aligned}
\frac{1}{W_{j, l}^{\text {near }}} \frac{\partial W_{j, l}^{\text {near }}}{\partial q^{0}}= & \frac{2(L+2)}{\sqrt{3}} \quad L:=\frac{2-l}{1-l} \\
\frac{1}{W_{j, l}^{\text {near }}} \frac{\partial W_{j, l}^{\text {near }}}{\partial q^{+}}= & \frac{2}{\sqrt{6}}\left[\mu_{1}^{L}\left(\mu_{2}^{2}+\mu_{3}^{2}\right)+\mu_{2}^{L}\left(\mu_{3}^{2}+\mu_{1}^{2}\right)+\mu_{3}^{L}\left(\mu_{1}^{2}+\mu_{2}^{2}\right)\right]^{-1}\left[2(L-1) \mu_{1}^{L}\left(\mu_{2}^{2}+\mu_{3}^{2}\right)\right. \\
& \left.+\mu_{2}^{L}\left(-(L+2) \mu_{3}^{2}+(4-L) \mu_{1}^{2}\right)+\mu_{3}^{L}\left((4-L) \mu_{1}^{2}-(L+2) \mu_{2}^{2}\right)\right] \\
\frac{1}{W_{j, l}^{\text {near }}} \frac{\partial W_{j, l}^{\text {near }}}{\partial q^{-}}= & \sqrt{2}\left[\mu_{1}^{L}\left(\mu_{2}^{2}+\mu_{3}^{2}\right)+\mu_{2}^{L}\left(\mu_{3}^{2}+\mu_{1}^{2}\right)+\mu_{3}^{L}\left(\mu_{1}^{2}+\mu_{2}^{2}\right)\right]^{-1}\left[2 \mu_{1}^{L}\left(\mu_{2}^{2}-\mu_{3}^{2}\right)\right. \\
& \left.+\mu_{2}^{L}\left((L-2) \mu_{3}^{2}+L \mu_{1}^{2}\right)-\mu_{3}^{L}\left(L \mu_{1}^{2}+(L-2) \mu_{2}^{2}\right)\right]
\end{aligned}
$$

These now have to be evaluated on the Kasner solution.

Observe that the logarithmic derivatives of the potential with respect to $q^{ \pm}$are ratios of homogeneous polynomials of the same degree. Hence any common factors in $\mu_{I}$ will cancel out. From (65), it is obvious that effectively we can take,

$$
\mu_{1}:=\mu^{-3}, \mu_{2}:=\nu^{-1}, \mu_{3}:=\nu, \quad \text { where } \mu:=e^{\frac{q^{0}}{\sqrt{3}} \cos \phi}, \nu:=e^{q^{0} \sin \phi} .
$$

The common factor canceled is $Q\left(q^{0}, j\right) \mu$. Substituting these in (70, 171) leads to,

$$
\begin{aligned}
& \frac{1}{W_{j, l}^{\text {near }}} \frac{\partial W_{j, l}^{\text {near }}}{\partial q^{+}}=\frac{2}{\sqrt{6}}\left[\mu^{-3 L}\left(\nu^{2}+\nu^{-2}\right)+\mu^{-6}\left(\nu^{L}+\nu^{-L}\right)+\left(\nu^{L-2}+\nu^{-(L-2)}\right)\right]^{-1} \\
& {\left[2(L-1) \mu^{-3 L}\left(\nu^{2}+\nu^{-2}\right)+(4-L) \mu^{-6}\left(\nu^{L}+\nu^{-L}\right)-\right.} \\
& \left.(L+2)\left(\nu^{L-2}+\nu^{-(L-2)}\right)\right] \\
& =: \frac{2}{\sqrt{6}} D^{-1} a_{+}
\end{aligned}
$$




$$
\begin{aligned}
& \frac{1}{W_{j, l}^{\text {near }}} \frac{\partial W_{j, l}^{\text {near }}}{\partial q^{-}}=-\sqrt{2}\left[\mu^{-3 L}\left(\nu^{2}+\nu^{-2}\right)+\mu^{-6}\left(\nu^{L}+\nu^{-L}\right)+\left(\nu^{L-2}+\nu^{-(L-2)}\right)\right]^{-1} \\
& {\left[2 \mu^{-3 L}\left(\nu^{2}-\nu^{-2}\right)+L \mu^{-6}\left(\nu^{L}-\nu^{-L}\right)+(L-2)\left(\nu^{L-2}-\nu^{-(L-2)}\right)\right]} \\
& =:-\frac{2}{\sqrt{6}} D^{-1} a_{-} \\
& \kappa^{+}=\frac{2(L+2)}{\sqrt{3}} \cos \phi-\frac{1}{\sqrt{3}} \frac{a_{+}}{D} \\
& \kappa^{-}=\frac{2(L+2)}{\sqrt{3}} \sin \phi+\frac{1}{\sqrt{3}} \frac{a_{-}}{D} \\
& \lambda=\frac{1}{\sqrt{3}}\left[2(L+2)-\frac{a_{+} \cos \phi}{D}+\frac{a_{-} \sin \phi}{D}\right\}
\end{aligned}
$$

The eigenvalue $\lambda$ is a function of $\phi$ and $q^{0}$. Generic stability of Kasner solution requires $\lambda$ to be positive for all $\phi$ and at every $q^{0}$ less than the initial $q^{0}$. A necessary condition for this to be true is that $a_{+}^{2}+a_{-}^{2}<4(L+2)^{2} D^{2}$. This is easily checked independently of $\phi, q^{0}$, completing the proof that the Kasner solution is stable under inclusion of the effective potential for all $\phi$ and for all $q^{0}$ less than the initially chosen $q^{0}$.

An identical calculation can be repeated for the classical potential by using the far form of the potential (which is independent of $l$, of course). However, now $\lambda$ does not have $a$ definite sign for all $\phi, q^{0}$. For a fixed $\phi, \lambda$ changes sign. The positive sign corresponds to 'Kasner epochs' while the negative sign corresponds to 'replacement of Kasner epochs'.

\section{Relation to Kasner Sphere}

It is conventional to analyze the asymptotic behavior of the approach to a singularity in terms of the scale factors of the classical geometry. We can obtain such a description by noting that (all scale factors, triad components positive),

$$
\begin{aligned}
& p^{I}=a_{J} a_{K} \\
& a_{I}=\frac{\text { volume }}{p^{I}}=(2 j)^{\frac{1}{2}} \frac{Q^{3 / 2}}{\mu_{I}} \quad \text { i.e. } \\
& a_{1}=e^{\frac{q^{0}}{\sqrt{3}}} e^{-\frac{4}{\sqrt{6}} R \cos \theta} \\
& a_{2}=e^{\frac{q^{0}}{\sqrt{3}}} e^{+\frac{2}{\sqrt{6}} R \cos \theta(1-\sqrt{3} \tan \theta)} \\
& a_{3}=e^{\frac{q^{0}}{\sqrt{3}}} e^{+\frac{2}{\sqrt{6}} R \cos \theta(1+\sqrt{3} \tan \theta)}
\end{aligned}
$$

For the Kasner solution, $R\left(q^{0}\right)=-\frac{1}{\sqrt{2}} q^{0}, \theta=\theta_{0}$ while the standard parameterization is $a_{I} \sim \mathrm{t}^{2 \alpha_{I}}=e^{2 \alpha_{I} \ln \mathrm{t}}$ such that $\sum_{I} \alpha_{I}=1=\sum_{I} \alpha_{I}^{2}$ ('Kasner sphere'). Putting $q^{0}=2 \zeta \ln \mathrm{t}$, 
comparison implies,

$$
\begin{aligned}
& \alpha_{1}=\frac{\zeta}{\sqrt{3}}\left\{1+2 \cos \theta_{0}\right\} \\
& \alpha_{2}=\frac{\zeta}{\sqrt{3}}\left\{1-\cos \theta_{0}\left(1-\sqrt{3} \tan \theta_{0}\right)\right\} \\
& \alpha_{3}=\frac{\zeta}{\sqrt{3}}\left\{1-\cos \theta_{0}\left(1+\sqrt{3} \tan \theta_{0}\right)\right\}
\end{aligned}
$$

The Kasner Sphere conditions are then automatically satisfied with the choice $\zeta=\frac{1}{\sqrt{3}}$ which identifies the co-moving time $\mathrm{t}$ as $e^{\frac{\sqrt{3}}{2} q^{0}}$ which is consistent with the definition of $q^{0}$.

It is obvious now that a point on the Kasner sphere corresponds to a choice of $\theta_{0}$. Oscillatory behavior corresponds to a motion of the system point passing through different angular sectors mentioned above.

Observe that a deviation from Kasner solution ( $\theta$ non-constant) does not imply oscillatory behavior. The $\theta$ motion may still be confined to one of the angular sectors leading to a Kasner-like behavior. For the classical potential with its confining nature (infinite walls), non-constant $\theta$ implies traversing all the angular sectors and thus oscillatory behavior. For the modified potential such an implication is not automatic although conceivable.

In the next section on the qualitative analysis we will see that the asymptotic solution is indeed a Kasner solution.

\section{Domain of Validity}

Before we consider the qualitative analysis, it is useful to make explicit the domain of validity of the effective Hamiltonian. Recall that the derivation of the effective Hamiltonian is based on the validity of the continuum approximation and the validity of the WKB approximation.

The continuum approximation itself is valid for $m_{I} \gg 1$ which implies $\mu_{I} \gg \epsilon:=1 /(2 j)$. In terms of the $q^{ \pm, 0}$ these restrictions translate to:

$$
\begin{aligned}
Q \gg \epsilon & \Leftrightarrow \quad q^{0}>0 ; \\
\frac{\sqrt{6}}{4} \ln (\epsilon / Q) & \ll q^{+} \ll \frac{\sqrt{6}}{2} \ln (Q / \epsilon) ; \\
-\frac{1}{\sqrt{3}}\left(\frac{\sqrt{6}}{2} \ln (Q / \epsilon)-q^{+}\right) & \ll q^{-} \ll \frac{1}{\sqrt{3}}\left(\frac{\sqrt{6}}{2} \ln (Q / \epsilon)-q^{+}\right) .
\end{aligned}
$$


Equivalently, using $Q / \epsilon=\mathrm{Vol}^{2 / 3}$ we have,

$$
\begin{aligned}
& \mathrm{Vol} \gg 1 \\
& -\frac{1}{\sqrt{6}} \ln (\mathrm{Vol}) \ll q^{+} \ll \sqrt{\frac{2}{3}} \ln (\mathrm{Vol}) ; \\
& -\frac{1}{\sqrt{3}}\left(\sqrt{\frac{2}{3}} \ln (\mathrm{Vol})-q^{+}\right) \ll q^{-} \ll \frac{1}{\sqrt{3}}\left(\sqrt{\frac{2}{3}} \ln (\mathrm{Vol})-q^{+}\right) \text {. }
\end{aligned}
$$

For a given $l$, the effective potential becomes negative for some $Q_{l}^{*}$, independently of $j$ and the corresponding $\operatorname{vol}_{l}^{*} \approx\left(2 j Q_{l}^{*}\right)^{\frac{3}{2}}$. To be consistent with the domain of validity, $\operatorname{Vol}_{l}^{*}>1$ must hold which can be arranged by choosing $j$ sufficiently large. Having chosen $\mathrm{Vol}_{l}^{*}$, the limits on $q^{ \pm}$follow.

Some typical values are: $Q_{0.1}^{*} \approx 0.91 ; Q_{0.5}^{*} \approx 1.086 ; Q_{0.9}^{*} \approx 1.38$. Clearly, for smaller $l$ we need to choose a larger $j$ in order to stay within the domain of validity. (In this context it is interesting to note that both conceptual considerations in the full theory and phenomenological studies in the context of early universe inflation point to values $j>1 / 2$ 35].) Thus we see that disappearance of the walls can be seen generically and reliably within the effective Hamiltonian picture. For the plots shown in [22], the ranges of $q^{ \pm}$are in the domain of validity.

For the stability analysis above, for large enough $j$, we can have all $\mu_{I} \ll 1$ even though $q^{0}>0$ justifying the use of the near form of the potential. The Kasner stability is thus seen staying consistent with the validity of the effective Hamiltonian.

One reason for this demarcation is that for any given fixed volume, the large $R$ form of the effective potential is very different from that of the classical potential. In particular, the classical potential is always large and positive while the effective one is large and negative. Only the inner side of the walls of the effective potential 'matches' with the classical potential for large volume. Consequently, the 'initial conditions' for the internal dynamics can be either in the inner side of the walls or the outer side of the walls - an option not available for the classical potential.

In the next section, we will go beyond the domain of validity by considering the limit $\mathrm{Vol} \rightarrow 0$. 


\section{QUALITATIVE ANALYSIS}

In this section we will pretend that the effective description is valid all the way to zero volume or $q^{0} \rightarrow-\infty$. Although we know that this cannot be true, we are considering this by way of contrasting the dynamics implied by the classical potential. We will therefore be interested in the asymptotic behavior as $q^{0} \rightarrow-\infty$ of the trajectories in the anisotropy plane. This analysis proceeds in stages. Asymptotically one of the following two possibilities can happen: either $R\left(q^{0}\right)$ remains bounded or it does not. In the first stage, we will first rule out a bounded asymptotic motion while in the second stage we will show that asymptotic motion always approaches a Kasner trajectory.

Proposition 1 Asymptotically, $R\left(q^{0}\right)$ cannot remain bounded.

The proof is by contradiction. Assume that the asymptotic motion is bounded. Then all the $\mu_{I}$ must become vanishingly small due to the explicit exponential $q^{0}$ dependence. Thus for sufficiently early time (small volume), we can use the near form of potential valid for $\mu_{I} \ll 1$.

Consider now just the radial motion, $R\left(q^{0}\right)$. Since it is bounded, it can either tend to a limiting value $R_{*}$ or it will keep oscillating. If it has a limiting value, then we must have $\dot{R}, \ddot{R}=0$ at $R=R_{*}$. However, for the near form of the potential, its radial derivative is negative. The radial equation of motion (52) then implies a contradiction unless possibly $R_{*}=0$.

For $R \sim 0$, we have,

$$
W_{j, l}^{\text {near }}(R, \theta) \approx-2(2 j)^{-\frac{3-l}{1-l}}\left(\frac{3}{1+l}\right)^{\frac{1}{1-l}} e^{\frac{2}{\sqrt{3}}\left(\frac{4-3 l}{1-l}\right) q^{0}}\left(3+\frac{8-12 l+6 l^{2}}{(1-l)^{2}} R^{2}\right) .
$$

Since at $R=0$ the potential is negative and $h^{-1}$ must be strictly positive, we must have $\left(\dot{R}^{2}+R^{2} \dot{\theta}^{2}\right)>1 / 2$. But this is impossible at $R_{*}=0$. Thus, $R\left(q^{0}\right)$ cannot have any finite limiting value.

If $R\left(q^{0}\right)$ oscillates, then it must have several extrema. Now the radial equation (52) together with the fact that $\frac{\partial W_{j, l}^{\text {near }}}{\partial R}<0$ implies that $\ddot{R}$ must be positive at any extremum. Thus the radial motion can have at the most one minimum and oscillatory motion is ruled out. Since both the oscillatory and the limiting radial motions are ruled out, we conclude that the trajectory cannot be confined to a bounded region and the proof is completed. 
For analyzing the case of unbounded motion, it is convenient to use a vector notation:

$$
\begin{aligned}
& \mu_{I}:=\frac{1}{2 j} e^{\rho_{I} q^{0}+\vec{\sigma}_{I} \cdot \vec{q}}, \quad \rho_{I}=\frac{2}{\sqrt{3}}=: \rho_{0} \quad \forall \mathrm{I}=1,2,3 \\
& \vec{\sigma}_{1}:=\left(\frac{4}{\sqrt{6}}, 0\right), \vec{\sigma}_{2}:=\left(-\frac{2}{\sqrt{6}}, \sqrt{2}\right), \vec{\sigma}_{3}:=\left(-\frac{2}{\sqrt{6}},-\sqrt{2}\right) \\
& \vec{\sigma}_{I}^{2}=\frac{8}{3}, 2 \rho_{0}^{2}=\sigma_{I}^{2}, \quad \sum_{I} \vec{\sigma}_{I}=0
\end{aligned}
$$

In addition, we also have the expression for $\mu_{I}$ in terms of $R, \theta$ given in equation (56).

Observe that the ratios of the $\mu_{I}$ 's do not have any explicit dependence on $q^{0}$ and since $R\left(q^{0}\right)$ is very large, these ratios either diverge, vanish or become equal to 1 (the last case only along three special directions). Values of individual $\mu_{I}$ 's however depend on the relative growth of $R\left(q^{0}\right)$ and $\left|q^{0}\right|$. We will say: $R\left(q^{0}\right)$ is dominant if $\frac{R\left(q^{0}\right)}{\left|q^{0}\right|} \rightarrow \infty$, sub-dominant if $\frac{R\left(q^{0}\right)}{\left|q^{0}\right|} \rightarrow 0$ and marginal if $\frac{R\left(q^{0}\right)}{\left|q^{0}\right|} \rightarrow \frac{\xi}{\sqrt{2}}$, where $\xi>0$ is constant such that $\xi=1$ corresponds to a Kasner motion. For sub-dominant $R\left(q^{0}\right)$ and marginal one with $\xi \leq 1$, all $\mu_{I}$ 's vanish (generically in the marginal case) while for dominant and $\xi>1$ cases, two of the $\mu_{I}$ 's diverge (vanish) while the third one vanishes (diverges) since the product of all $\mu_{I}$ 's vanishes. Since $2 j>1$, all $\mu_{I}$ are either much smaller or larger than 1 so that the corresponding forms of the $F\left(\mu_{I}\right)$ can be used to approximate the effective potential. To get explicit forms, we have to consider various cases. Noting that the potential is invariant under cyclic permutation of the $\mu_{I}$, it suffices to consider the cases: $(I) \mu_{3} \ll \mu_{2} \ll \mu_{1}$ and $\left(I^{\prime}\right) \mu_{2} \ll \mu_{3} \ll \mu_{1}$. It is easy to see that $(I)$ corresponds to large $R$ and $\overrightarrow{\sigma_{3}} \cdot \vec{q}<\overrightarrow{\sigma_{2}} \cdot \vec{q}<\overrightarrow{\sigma_{1}} \cdot \vec{q}$ from which it follows that $\vec{q}$ must be confined to $0<\theta<\frac{\pi}{3}$. Likewise, the region $\left(I^{\prime}\right)$ corresponds to large $R$ and $-\frac{\pi}{3}<\theta<0$ (see figure प).

Within these regions (ordering of the $\mu_{I}$ 's), we have further sub-cases,

$$
\text { (a) } \mu_{3} \ll \mu_{2} \ll \mu_{1} \ll 1 \text {, (b) } \mu_{3} \ll \mu_{2} \ll 1 \ll \mu_{1} \text {, (c) } \mu_{3} \ll 1 \ll \mu_{2} \ll \mu_{1} \text {. }
$$

The case where all $1 \ll \mu_{I}$, would imply that their product is larger than 1 which is not possible for $q^{0} \rightarrow-\infty$.

For these cases, we will derive the asymptotic form of the potential and analyze the equations to deduce that only marginal $R\left(q^{0}\right)$ is possible with $\xi=1$.

To derive the asymptotic potential, we prove a couple of lemmas:

Lemma 1 If $R\left(q^{0}\right) \rightarrow \infty$ as $q^{0} \rightarrow-\infty$ then for any real numbers $l, m, n,\left(\mu_{I}\right)^{l}\left(\mu_{J}\right)^{m}\left(\mu_{K}\right)^{n}$ either diverges, vanishes or goes to $(2 j)^{-(l+m+n)}$. 


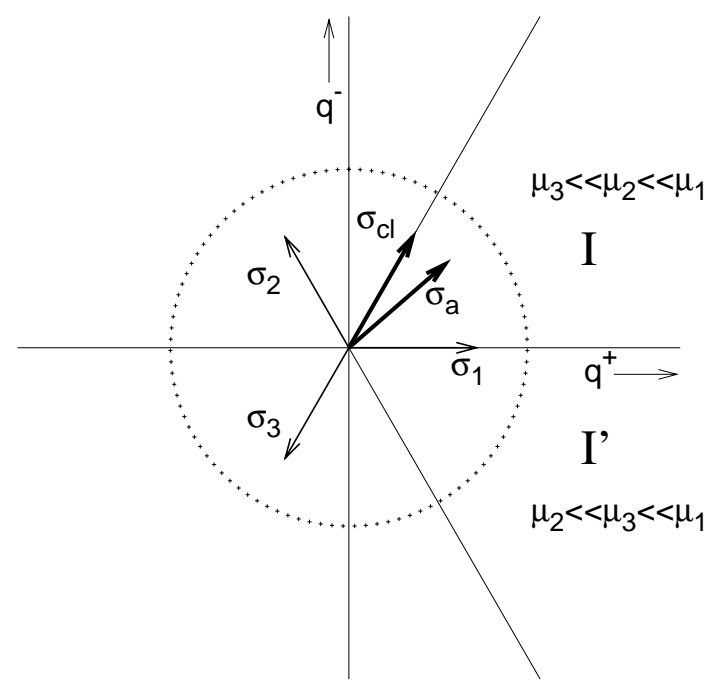

FIG. 1: The asymptotic regions I and $\mathrm{I}^{\prime}$ of the anisotropy plane. $R$ is outside the circle. $\vec{\sigma}_{a}$ varies with the ambiguity parameter $l$ staying within I. The vectors shown are unit vectors.

The proof is obvious since $\mu_{I} \sim e^{\frac{2}{\sqrt{3}} q^{0} R\left(q^{0}\right) \cos \theta_{I}\left(q^{0}\right)}$ and asymptotically, the exponent is either positive, negative or zero.

Lemma 2 The spin connection components obey the inequalities:

(a) $1 \gg \mu_{I} \gg \mu_{J} \gg \mu_{K} \Rightarrow \Gamma_{K} \gg \Gamma_{J} \gg \Gamma_{I}, \Gamma_{K} \gg \Gamma_{J} \Gamma_{I}$

(b) $\mu_{I} \gg 1 \gg \mu_{J} \gg \mu_{K} \Rightarrow \Gamma_{K} \gg \Gamma_{J}, \Gamma_{K} \gg \Gamma_{I}, \Gamma_{K} \gg \Gamma_{J} \Gamma_{I}$;

(c) $\mu_{I} \gg \mu_{J} \gg 1 \gg \mu_{K} \Rightarrow \Gamma_{K} \gg \Gamma_{J} \gg \Gamma_{I}, \Gamma_{K} \gg \Gamma_{J} \Gamma_{I}$.

To prove the above lemma, it should be noted that the ratio of any two spin connections, say $\Gamma_{I}$ and $\Gamma_{J}$ can be written as

$$
\frac{\Gamma_{I}}{\Gamma_{J}}=\left(\frac{\mu_{J}}{\mu_{I}}\right)\left[\frac{\frac{F\left(\mu_{K}\right)}{\mu_{K}}+\frac{F\left(\mu_{J}\right)}{\mu_{J}}-F\left(\mu_{I}\right)^{2}}{\frac{F\left(\mu_{K}\right)}{\mu_{K}}+\frac{F\left(\mu_{I}\right)}{\mu_{I}}-F\left(\mu_{J}\right)^{2}}\right],
$$

where the term inside the parenthesis is explicitly in reverse order. Also it can be shown that for all cases and for all allowed values of $l$, the term inside the bracket asymptotically either vanishes or goes to a constant or diverges but the divergence is weaker than the convergence of $\left(\frac{\mu_{J}}{\mu_{I}}\right)$. 
It follows that the potential takes the form $W_{j, l}(\vec{\mu}) \approx-8 j^{2} \mu_{I} \mu_{J} \Gamma_{K}$. From Lemma 11 it follows that $\Gamma_{K}$ will become a monomial in the $\mu$ 's. The potential then takes the form:

$$
\begin{aligned}
& W_{j, l}^{(a)}(\vec{\mu}) \simeq-4 j^{2}\left(\frac{3}{l+1}\right)^{\frac{1}{1-l}} \mu_{I}^{\frac{2-l}{1-l}} \mu_{J}^{2}, \\
& W_{j, l}^{(b)}(\vec{\mu}) \simeq-4 j^{2}\left(\frac{3}{l+1}\right)^{\frac{1}{1-l}} \mu_{I}^{2} \mu_{J}^{\frac{2-l}{1-l}} \\
& \text { or }-4 j^{2} \mu_{J}^{2} \\
& \text { or }-4 j^{2}\left\{1+\left(\frac{3}{1+l}\right)^{\frac{1}{1-l}}(2 j)^{-\frac{2-l}{1-l}}\right\} \mu_{J}{ }^{2}, \\
& W_{j, l}^{(c)}(\vec{\mu}) \simeq-4 j^{2} \mu_{I}^{2} .
\end{aligned}
$$

Thus we have proved,

Proposition 2 In all cases where the $\mu_{I}$ either vanish or diverge, the potential necessarily takes the form,

$$
W_{j, l}\left(R, \theta, q^{0}\right) \sim-C e^{\rho q^{0}+\vec{\sigma} \cdot \vec{q}}
$$

where $C, \rho, \vec{\sigma}$ are constants with $C>0, \rho>0$ and $\vec{\sigma}^{2} \leq 2 \rho^{2}$.

Thus in all the cases, the potential has the form (94). In fact, for the sub-case (a) and the first of the sub-case (b) above, we have $\vec{\sigma}^{2} /\left(2 \rho^{2}\right)=\left(3 l^{2}-6 l+4\right) /(3 l-4)^{2}$, which is smaller than one for all $0<l<1$ (and exactly one for the limiting case $l=1$ ). In the remaining two of the sub-case (b) and the sub-case (c), we have $\vec{\sigma}^{2} /\left(2 \rho^{2}\right)=1$. Notice that the asymptotic potential is negative. Together with $h>0$, this implies that $1-2 \dot{\vec{q}}<0$.

Using the asymptotic form of the potential, the Cartesian form (47) of the equations becomes $(\vec{v}:=\dot{\vec{q}})$,

$$
\dot{\vec{v}}=\left(\vec{v}^{2}-\frac{1}{2}\right)\left(\frac{\vec{\sigma}}{2}+\rho \vec{v}\right) \quad, \quad \vec{v}^{2}>\frac{1}{2}, 2 \rho^{2}-\vec{\sigma}^{2} \geq 0 .
$$

Notice that the above equation is a first order, autonomous equation for the velocity. The (vector) equation has fixed points at $\vec{v}^{2}=\frac{1}{2}$ and at $\vec{v}=-\frac{\vec{\sigma}}{2 \rho}$. For the latter, isolated fixed point, $\vec{v}^{2}=\frac{1}{2} \frac{\sigma^{2}}{2 \rho^{2}} \leq \frac{1}{2}$ and hence this is not accessible.

Taking scalar product of the equation (95) with $2 \vec{v}$ one gets,

$$
\dot{\vec{v}}^{2}=\left(\vec{v}^{2}-\frac{1}{2}\right)\left(2 \rho \vec{v}^{2}\right)\left(\frac{\vec{\sigma} \cdot \vec{v}}{2 \rho \vec{v}^{2}}+1\right) .
$$

The inequalities on the $\vec{v}^{2}$ and $\vec{\sigma}^{2}, \rho^{2}$ imply that the third factor is always positive and hence $\vec{v}^{2}$ must decrease as $q^{0}$ is decreased. Clearly, $\vec{v}^{2}$ is bounded above by $\left(\vec{v}_{\text {initial }}\right)^{2}$ and 
bounded below by $\frac{1}{2}$. Since the equation for $\vec{v}^{2}$ is monotonic, asymptotically $\vec{v}^{2}$ must reach the lower bound.

Taking the 'cross product' of the equation (95) with $\vec{\sigma}$ one obtains,

$$
(\vec{\sigma} \times \vec{v})=\rho\left(\vec{v}^{2}-\frac{1}{2}\right)(\vec{\sigma} \times \vec{v})
$$

This equation shows that either $\vec{\sigma} \dot{\times} \vec{v}=0$ or, if non-zero, cannot change sign. If $\phi$ denotes the angle between $\vec{v}$ and $\vec{\sigma}$, then the cross product is $v \sigma \sin \phi(v:=|\vec{v}|, \sigma:=|\vec{\sigma}|)$ which is clearly bounded. Hence it must also approach a limiting value. Since both the magnitude of the velocity as well as its component orthogonal to $\vec{\sigma}$ approach a limiting value, we conclude that asymptotically $\vec{v}$ must approach some $\vec{v}_{*}$ such that $\vec{v}_{*}^{2}=\frac{1}{2}$. This also means that $R\left(q^{0}\right)$ must be marginal with $\xi=1$, both the dominant and the sub-dominant as well as marginal with $\xi \neq 1$ possibilities for $R\left(q^{0}\right)$ being ruled out. Since in the sub-cases listed in equation (89), (b) and (c) have at least one of $\mu_{1}, \mu_{2}$ to be larger than 1 and for $\xi=1$ marginal behavior all $\mu_{I}$ 's must be less than 1, these two sub-cases are ruled out. Thus, sub-case (a) is the only relevant case and, corresponding to region (I), we have $\vec{\sigma}=\vec{\sigma}_{a}$ which is read off from equation (91) as,

$$
\vec{\sigma}_{a}=\frac{2-l}{1-l} \vec{\sigma}_{1}+2 \vec{\sigma}_{2}
$$

As shown in the figure 1, $\vec{\sigma}_{a}$ lies within the region (I).

To study the nature of a candidate fixed point $\vec{v}_{*}$ of equation (95), linearize the equation by putting $\vec{v}=\vec{v}_{*}+\delta \vec{v}$. This leads to,

$$
\dot{\overrightarrow{\delta v}}=\left(\vec{v}_{*} \cdot \overrightarrow{\delta v}\right) \vec{\Delta} \quad, \quad \vec{\Delta}:=\vec{\sigma}+2 \rho \vec{v}_{*}
$$

Clearly, $\overrightarrow{\delta v}$ orthogonal to $\vec{v}_{*}$ is a zero mode of the linearized equation which does not evolve. Taking scalar product with $\vec{v}_{*}$ gives, using $\vec{v}_{*}^{2}=\frac{1}{2}$,

$$
\left(\vec{v}_{*} \cdot \overrightarrow{\delta v}\right)=\left(\vec{v}_{*} \cdot \overrightarrow{\delta v}\right)\left(\vec{v}_{*} \cdot \vec{\sigma}+\rho\right)
$$

Linear stability of $\vec{v}_{*}$ thus demands $\rho+\sigma\left(\cos \phi_{*}\right) / \sqrt{2}>0$. Since $\sqrt{2} \rho / \sigma \geq 1$, this condition is satisfied for all $\phi_{*}$. Thus we have proved that the asymptotic velocity under the evolution equation (95) is a Kasner velocity and every Kasner velocity is admissible. Furthermore, this velocity is approached exponentially as implied by the stability analysis. 
One can also derive a relation between an initial velocity and the limiting velocity. Denote: $v_{\|}:=v \cos \phi, v_{\perp}:=v \sin \phi$. Taking scalar and cross product of equation (95) with $\vec{\sigma}$ gives,

$$
\dot{v}_{\|}=\left(\vec{v}^{2}-\frac{1}{2}\right)\left(\frac{\sigma}{2}+\rho v_{\|}\right), \dot{v}_{\perp}=\left(\vec{v}^{2}-\frac{1}{2}\right)\left(\rho v_{\perp}\right) .
$$

If $v_{\perp}=0$ initially, then it is identically zero and therefore $\phi_{*}=0$ or $\pi$ depending upon the sign of $v_{\|}$. The $v_{\|}$equation can be easily integrated but we do not need the explicit solution. If $v_{\perp} \neq 0$ initially then it must retain its sign. In this case taking the ratio $\dot{v}_{\|} / \dot{v}_{\perp}$ gives a differential equation for $v_{\|}$as a function of $v_{\perp}$ which can be immediately integrated to give an orbit equation,

$$
v_{\perp}=A\left(v_{\|}+\frac{\sigma}{2 \rho}\right)
$$

where $A$ is a constant of integration.

The orbit is a straight line in the velocity plane always passing through the point $v_{\|}=$ $-\frac{\sigma}{2 \rho}\left(>-\frac{1}{\sqrt{2}}\right), v_{\perp}=0$ and with a slope determined by $A$. This point is exactly the isolated fixed point of the (95) equation.

Note that since $v_{\perp} \neq 0$ neither $A$ nor $\left(v_{\|}+\frac{\sigma}{2 \rho}\right)$ can be zero for any finite $q^{0}$. We also know that asymptotically $v_{\perp} \rightarrow v_{\perp *}:=\frac{1}{\sqrt{2}} \sin \phi_{*}$ and $v_{\|} \rightarrow v_{\| *}:=\frac{1}{\sqrt{2}} \cos \phi_{*}$. As noted earlier, only $\vec{\sigma}_{a}$ is consistent with asymptotic Kasner motion and for this case $2 \rho_{a}^{2}-\sigma_{a}^{2}$ is strictly positive. This implies that $\left(v_{\| *}+\frac{\sigma}{2 \rho}\right)$ is non-zero and therefore $v_{\perp *}$ is also non-zero. Evaluating equation (102) for initial values of $\left(v_{\|}, v_{\perp}\right)$ determines $A$ as a function of $v, \phi$ while evaluating the same equation for asymptotic values $\left(v_{\| *}, v_{\perp *}\right)$ determines the angle $\phi_{*}$. Explicitly, in terms of $A$,

$$
\begin{aligned}
& \cos \phi_{*}=\frac{1}{1+A^{2}}\left\{-A^{2} \frac{\sigma}{\sqrt{2} \rho} \pm \sqrt{1+A^{2}-A^{2} \frac{\sigma^{2}}{2 \rho^{2}}}\right\} \\
& \sin \phi_{*}=\frac{A}{1+A^{2}}\left\{\frac{\sigma}{\sqrt{2} \rho} \pm \sqrt{1+A^{2}-A^{2} \frac{\sigma^{2}}{2 \rho^{2}}}\right\}
\end{aligned}
$$

The two roots ( \pm signs) for $\sin \left(\phi_{*}\right)$ always have opposite signs and since the $\sin (\phi)$ does not change sign, the initial condition sets the sign and selects a unique limiting value $\phi_{*}$.

Now the trajectory in the anisotropy plane can be obtained trivially as, $\vec{q}\left(q^{0}\right)=\vec{v}_{*} q^{0}+\hat{q}$ which leads to,

$$
R\left(q^{0}\right) \sim-\frac{1}{\sqrt{2}} q^{0}+O(1) \quad, \quad \theta\left(q^{0}\right) \sim \theta_{0}+O\left(\frac{1}{\sqrt{-q^{0}}}\right),
$$


where $\theta_{0}=\phi_{*}$ plus the angle $\vec{\sigma}$ makes with the $q^{+}$axis. Notice that asymptotically $\vec{q}$ points in the direction opposite to that of $\vec{v}_{*}$.

Let us now compare a similar analysis for the classical potential. In the classical case, one does not need to distinguish the sub-cases of (89) as the potential is directly given as a sum of monomials in $\mu_{I}$. The asymptotic form in region (I) is the same as given in Proposition 2 with $C<0, \rho=\frac{4}{\sqrt{3}}, \vec{\sigma}=-4 \overrightarrow{\sigma_{3}}=\left(\frac{8}{\sqrt{6}}, 4 \sqrt{2}\right)$. This implies two differences compared to the effective case: $\frac{\sqrt{2} \rho}{\sigma}=\frac{1}{2}<1$ and $\vec{v}^{2}<\frac{1}{2}$. The first order, autonomous equation for the velocity remains the same as (95) (except for the inequalities) and so do the equations (96, 97). The isolated fixed point of (95) gets ruled out as before. Unlike the effective potential case, the third factor in (96) no longer has a fixed sign. If it is zero, then $v^{2}$ gets frozen at a fixed value and if it is non-zero, it cannot change its sign. Since the evolution of $v^{2}$ is bounded, once again we get that $v^{2} \rightarrow v_{*}^{2}$ although we cannot conclude at this stage that $v_{*}^{2}=\frac{1}{2}$. As before, $v \sin \phi$ is bounded and evolves monotonically thereby reaching some limiting value. Thus we conclude that $\vec{v} \rightarrow \vec{v}_{*}$ as before though without the condition $v_{*}^{2}=\frac{1}{2}$.

Properties of a candidate limiting velocity are analyzed by linearization, $\vec{v}=\vec{v}_{*}+\delta \vec{v}$. This leads to,

$$
\dot{\overrightarrow{\delta v}}=\gamma \vec{\Delta}+\gamma \rho \delta \vec{v}+\left(\vec{v}_{*} \cdot \overrightarrow{\delta v}\right) \vec{\Delta} \quad, \quad \vec{\Delta}:=\vec{\sigma}+2 \rho \vec{v}_{*} \quad, \quad \gamma:=v_{*}^{2}-\frac{1}{2} .
$$

Clearly, $\delta \vec{v} \rightarrow 0$ must hold and therefore either $\gamma=0$ or $\vec{\Delta}=\overrightarrow{0} . \vec{\Delta}=\overrightarrow{0}$ is ruled out since this gives $v_{*}^{2}=2$ and hence $\gamma=0$ must hold. We have recovered the $v_{*}^{2}=\frac{1}{2}$. The rest of the stability analysis now gives, exactly as before $\left(\rho+\frac{\sigma \cos \phi_{*}}{\sqrt{2}}\right)>0$ or $\cos \left(\phi_{*}\right)>-\frac{1}{2}$. Unlike the effective potential case, now we have a restriction on the asymptotic angles $\phi_{*}$. The angle $\phi_{*}$ can again be obtained as in eq. (103), however due to $\frac{\sigma^{2}}{2 \rho^{2}}=4$, the square roots are real only if $A^{2} \leq \frac{1}{3}$.

The orbit equation provided a very simple pictorial representation shown in Figure 2, The two differences between the effective potential and the classical potential control the location of the isolated fixed point $\left(v_{\perp}=0\right)$ and the setting of initial conditions. For both potentials the continuum of fixed points is determined by the circle: $v_{*}^{2}=\frac{1}{2}$. For the effective potential, the isolated fixed point lies inside the circle while the initial condition must be set outside the circle. For the classical case it is exactly the opposite. The monotonic decrease of $v^{2}$ in the case of the effective potential and monotonic increase of $v_{\|}$in the case of the classical potential shows the direction of traversal of the orbits. The exponential approach 
to $\vec{v}_{*}$ follows from the linear stability analysis.

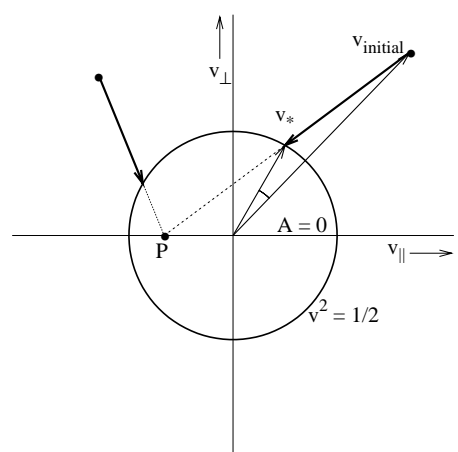

(a)

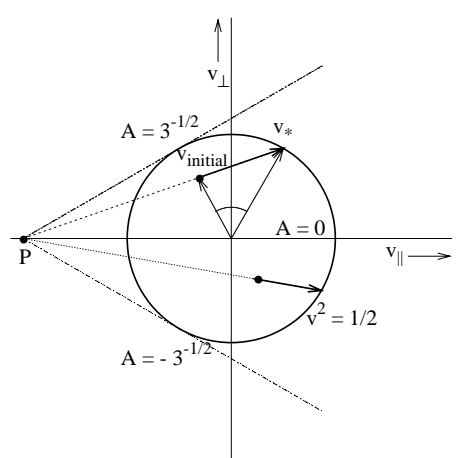

(b)

FIG. 2: The point $P$ and the circle constitute the fixed points. The heavy, directed straight line segments are typical orbits of the velocity dynamics in the velocity plane. The $v_{\|}, v_{\perp}$ are relative to $\vec{\sigma}$. The angle shown is the total change in the direction of the velocity vector. The figure (a) corresponds to the effective potential while (b) corresponds to the classical potential.

How does one understand the asymptotic Kasner solutions for the classical potential given the well known oscillatory behavior? Recall that the asymptotic direction reached in the anisotropy plane is opposite to that of $\vec{v}_{*}$ (since $q^{0}$ is negative). The stable Kasner velocity directions are confined to $-\frac{2 \pi}{3}$ to $\frac{2 \pi}{3}$ relative to $\vec{\sigma}$ which makes an angle $\frac{\pi}{3}$ to the $q^{+}$axis. The corresponding trajectories therefore satisfy $\frac{2 \pi}{3}<\theta_{0}<2 \pi$ which precisely excludes the region (I)! The qualitative analysis rests on the assumption that (95) remains valid throughout the evolution. This assumption must fail in the case of the classical potential - a trajectory that enters an asymptotic region (I, I' or their $120^{\circ}$ rotations), must exit it in a finite $q^{0}$ interval. By contrast, for the effective potential, since all $\phi_{*}$ are stable, there always exist directions such that $\theta_{0}$ is in the asymptotic region. The remaining directions will again correspond to exiting the region only to eventually enter another region with compatible $\theta^{0}$.

Combining similar analyses of all other asymptotic regions we conclude that any trajectory of the effective dynamics will always go exponentially to a Kasner trajectory as volume goes to zero, with at the most finitely many inter-region traversals (oscillations). 


\section{SUMMARY AND DISCUSSION}

Bianchi IX models are well known to display the most complex behavior close to a homogeneous singularity but in addition, in the BKL scenario, play a pivotal role in suggesting a strongly spatially dependent structure of the spatial slice close to a generic, inhomogeneous cosmological singularity. The primary source of this picture is the chaotic behavior of the Bianchi IX dynamics which forces infinite fragmentation with no correlations among the fragments. Since chaotic behavior itself is a consequence of unbounded growth of the curvature invariants, i.e. the singularity, and since the loop quantization of diagonal, homogeneous models has been shown to be singularity free, it is natural to focus on the modification due to the loop quantization of the vacuum Bianchi IX model.

Since the chaotic dynamics is best understood in the classical setting, we worked with an effective classical dynamics from the underlying, singularity free quantum dynamics. This was done by exploiting the (always) available continuum approximation together with a WKB ansatz for the continuum wave function. The most significant, qualitatively distinct effects of the loop quantization got incorporated into the effective potential via the definition of inverse triad components together with the quantization ambiguity parameters $(j \geq$ $\left.\frac{1}{2}, 0<l<1\right)$. The effective potential has several qualitative differences from the classical potential. (i) Its volume dependence is not in a factorizable form which makes the dynamical equations explicitly 'time' $\left(q^{0}\right)$ dependent. (ii) It does have walls for large volume but unlike the classical potential these walls are not confining - they have a finite height and a finite extent. (iii) For smaller volume, these walls just disappear, the potential becomes negative everywhere and approaches zero in the zero volume limit. Qualitatively one expects a simpler, non-oscillatory, behavior asymptotically resembling Kasner behavior since the potential vanishes.

We presented the details of the effective dynamics at two levels. In section $\mathbf{Q}$, we stayed entirely within the domain of validity of the effective model and demonstrated the nonasymptotic stability of the Kasner motion to the inclusion of the effective potential. The vanishing of inverse triad eigenvalues for small triad eigenvalues was directly responsible for this stability. In section $\nabla \mathbb{1}$, we extrapolated the effective dynamics beyond its natural domain of validity into the small volume regime, much as one does in the usual classical analysis. We proved in detail that for every initial condition, after at most finitely many 
oscillations, the trajectory will diverge to infinite anisotropies as a Kasner motion. The classical case was also contrasted. All the proofs work uniformly for all the allowed values of the ambiguity parameters demonstrating the robustness of loop quantum effects.

To explore the possible implications of the results for the structure of inhomogeneous singularities, let us assume the validity of the BKL picture and divide up a spatial slice into 'cells' which are approximately homogeneous and have (initial) anisotropies parameterized by Bianchi IX. We may picture the so decomposed initial spatial slice as a 'sprinkling of points' in the Bianchi IX anisotropy plane, each point corresponding to a cell. The approach to an inhomogeneous singularity can now be viewed as evolution of the initial points under a Bianchi IX effective (or otherwise) dynamics.

When the evolution is chaotic (as in the classical case), two nearby points will diverge away, effectively loosing initial correlations implied by the smooth geometry of the slice. This needs further decomposition and so on. Asymptotically then there would be infinitely many points performing un-correlated motion in the anisotropy plane - i.e. a non-interacting two dimensional 'gas'.

When the chaotic motion is replaced by asymptotic Kasner evolution (as with the effective dynamics), the points will move in a simpler manner getting distributed into the various regions and after some initial crossings between regions they will remain confined to their respective regions. Their correlations, just before this stage, will not be washed away and we will have an 'interacting gas.' In both cases, all angular sectors are 'filled' suggesting that a sufficiently large class of inhomogeneities can be parameterized as a distribution of anisotropies to the cells. (The inhomogeneities would be arbitrary in the case of chaotic dynamics.)

Just for contrast assume that Bianchi IX dynamics is governed by the negative of the classical potential. Since the walls disappear, this is a trivial way of removing chaos (by hand). The stable fixed points of the asymptotic dynamics now include all those for the classical potential but in addition the isolated fixed point at $\sigma_{\perp}=0, \sigma_{\|}=-\frac{\sigma}{2 \rho}=-\sqrt{2}$ is also accessible. Now the asymptotic motion compatible with the region would be to the isolated fixed point. By permutation, one will have just three possible asymptotic velocities (along the dips in the classical potential). All the initially distributed points will cluster in these directions and the singularity will look essentially homogeneous.

We will now use these three scenarios, classical potential, negative classical potential, 
and effective potential, to shed light on the general structure of classical singularities. In the context of the approach to a classical singularity it has been observed [36] that the beginning and the end of a universe like ours appear very different from each other. Our observable part of the universe starts out very homogeneous and isotropic, but turns much more complicated in the future. Black holes form and their singularities make space more and more inhomogeneous. Let us now assume that the universe may re-collapse. Then by time reversal symmetry the structure of both the initial and the final singularity at the boundaries of space-time would have to be the same. If the universe does form black holes (which once formed will not disappear in a classical context), then the inhomogeneity implied by their presence will not disappear implying a strongly inhomogeneous structure for the final singularity. From what we can tell by observations of our own universe, then, the situation looks far from time reversal symmetric. Right now space is very homogeneous and isotropic at large scales, and is expected to have been even more homogeneous earlier, while we do not see a reason for it to homogenize away the structure implied by black holes in the future.

The large degree of homogeneity is usually explained by referring to inflation, which expands a small patch of an initial space and thus removes inhomogeneities. (However, whether inflation begins at all in an inhomogeneous context is apparently an unresolved issue [37, 38]. Moreover, if the classical BKL dynamics is true, this initial space close to the classical singularity would have structure at arbitrarily small scales which would be difficult to smooth out even for inflation. If the chaotic behavior leads to a fractal initial state, inhomogeneities would not be removed at all due to self-similarity.) If the inhomogeneous structure is determined by the chaotic Bianchi IX dynamics, then the primordial anisotropies would be completely arbitrary in such a patch. However, there are arguments to the effect that any such anisotropies would be washed away before inflation ends [39]. This would be good for phenomenology in that one does not have to worry about the details prior to the beginning of inflation but then one has also lost any contact with whatever quantum effects that would be present in the earlier epoch. Alternatively, those effects could be influential in determining the beginning of inflation itself. Deciding this may be harder when homogeneous patches are un-correlated. In any case, the classical expectation for the structure of singularities would be consistent with the expected inhomogeneous end, but could be problematic for the beginning. 
The hypothetical case of a negative classical potential considered above implies homogeneous structure for the singularity. This would be quite desirable for the initial singularity since it is compatible with the observed large scale homogeneity. One may at the most have to worry about the isotropization issue. However, it would be in conflict with an inhomogeneous final singularity and hence inconsistent with black hole formation during the evolution of the universe. Thus merely removing the chaotic Bianchi IX dynamics would not be satisfactory.

The effective dynamics, finally, offers a third possibility. It admits a sufficiently general class of inhomogeneities (thereby accommodating black hole formation) but does not give rise to structure on arbitrarily small scales and retains some correlations among the patches. This may make starting inflation easier. Alternatively the mechanism of inflation could be very different which will not only homogenize but may also retain some imprint of the underlying quantum dynamics. The consequences are not worked out at present but it does open up other possibilities to be explored. In this aspect, matter will almost certainly play a significant role. The influence of matter on the effective dynamics as well as the other issues mentioned above will be dealt with elsewhere.

While the effective dynamics is consistent with our expectations for both the beginning and the end of the universe, the apparent time reversal asymmetry remains. This is explained by the fact that the situation is, in fact, time asymmetric due to our own position in the universe. We can see only some part of it, not the whole space-time, and in particular we see only a small part of the beginning. With the current understanding, the observable part of our universe can well be part of a classical space-time with a very inhomogeneous initial singularity. Since most of the initial singularity is unobservable, however, it is not discussed further. The final singularity, on the other hand, is completely unobservable until it is reached. If we compare only observable properties from within the universe, we simply cannot possibly know enough to tell whether past and future singularities are similar. If we compare the theoretical structure of a space-time from outside, then we conclude that in fact there is no conceptual difference between the beginning and the end of a generic spacetime. Only if we compare the observable part of the initial singularity with the theoretical expectation for a final singularity does the time asymmetry appear. 


\section{Acknowledgments}

We thank A. Coley, R. Penrose, A. Rendall and R. Vaas for discussions.

[1] S. W. Hawking and G. F. R. Ellis, The Large Scale Structure of Space-Time, Cambridge University Press, 1973

[2] V. A. Belinskii, I. M. Khalatnikov, and E. M. Lifschitz, A general solution of the Einstein equations with a time singularity, Adv. Phys. 13 (1982) 639-667

[3] D. Garfinkle, Numerical simulations of generic singularities, gr-qc/0312117

[4] T. Damour, M. Henneaux, and H. Nicolai, Cosmological Billiards, Class. Quantum Grav. 20 (2003) R145-R200, hep-th/0212256

[5] T. Damour and M. Henneaux, Chaos in Superstring Cosmology, Phys. Rev. Lett. 85 (2000) 920-923

[6] A. Coley, No Chaos in Brane-World Cosmology, Class. Quantum Grav. 19 (2002) L45-L56, hep-th/0110117

[7] C. Rovelli, Loop Quantum Gravity, Living Reviews in Relativity 1 (1998) 1, gr-qc/9710008, http://www.livingreviews.org/Articles/Volume1/1998-1rovelli,

T. Thiemann, Introduction to Modern Canonical Quantum General Relativity, gr-qc/0110034;

A. Ashtekar and J. Lewandowski, Background Independent Quantum Gravity: A Status Report, gr-qc/0404018

[8] M. Bojowald and H. A. Morales-Técotl, Cosmological applications of loop quantum gravity, In Proceedings of the Fifth Mexican School (DGFM): The Early Universe and Observational Cosmology, Lect. Notes Phys. (in press), Springer-Verlag, 2004, gr-qc/0306008

[9] M. Bojowald, Loop Quantum Cosmology: II. Volume Operators, Class. Quantum Grav. 17 (2000) 1509-1526, gr-qc/9910104

[10] C. Rovelli and L. Smolin, Discreteness of Area and Volume in Quantum Gravity, Nucl. Phys. B 442 (1995) 593-619, gr-qc/9411005, Erratum: Nucl. Phys. B 456 (1995) 753

[11] A. Ashtekar and J. Lewandowski, Quantum Theory of Geometry I: Area Operators, Class. Quantum Grav. 14 (1997) A55-A82, gr-qc/9602046

[12] A. Ashtekar and J. Lewandowski, Quantum Theory of Geometry II: Volume Operators, Adv. 
Theor. Math. Phys. 1 (1997) 388-429, gr-qc/9711031

[13] M. Bojowald, Loop Quantum Cosmology III: Wheeler-DeWitt Operators, Class. Quantum Grav. 18 (2001) 1055-1070, gr-qc/0008052

[14] M. Bojowald, Loop Quantum Cosmology IV: Discrete Time Evolution, Class. Quantum Grav. 18 (2001) 1071-1088, gr-qc/0008053

[15] M. Bojowald, Inverse Scale Factor in Isotropic Quantum Geometry, Phys. Rev. D 64 (2001) 084018, gr-qc/0105067

[16] M. Bojowald, Homogeneous loop quantum cosmology, Class. Quantum Grav. 20 (2003) 25952615, gr-qc/0303073

[17] M. Bojowald, Isotropic Loop Quantum Cosmology, Class. Quantum Grav. 19 (2002) 27172741, gr-qc/0202077

[18] M. Bojowald, Absence of a Singularity in Loop Quantum Cosmology, Phys. Rev. Lett. 86 (2001) 5227-5230, gr-qc/0102069

[19] M. Bojowald, G. Date, and K. Vandersloot, Homogeneous loop quantum cosmology: The role of the spin connection, Class. Quantum Grav. 21 (2004) 1253-1278, gr-qc/0311004

[20] M. Bojowald, The Semiclassical Limit of Loop Quantum Cosmology, Class. Quantum Grav. 18 (2001) L109-L116, gr-qc/0105113

[21] M. Bojowald and G. Date, Consistency conditions for fundamentally discrete theories, Class. Quantum Grav. 21 (2004) 121-143, gr-qc/0307083

[22] M. Bojowald and G. Date, Quantum suppression of the generic chaotic behavior close to cosmological singularities, Phys. Rev. Lett. 92 (2004) 071302, gr-qc/0311003

[23] M. Bojowald and H. A. Kastrup, Symmetry Reduction for Quantized Diffeomorphism Invariant Theories of Connections, Class. Quantum Grav. 17 (2000) 3009-3043, hep-th/9907042

[24] A. Ashtekar, J. C. Baez, A. Corichi, and K. Krasnov, Quantum Geometry and Black Hole Entropy, Phys. Rev. Lett. 80 (1998) 904-907, gr-qc/9710007

[25] A. Ashtekar, J. C. Baez, and K. Krasnov, Quantum Geometry of Isolated Horizons and Black Hole Entropy, Adv. Theor. Math. Phys. 4 (2001) 1-94, gr-qc/0005126

[26] C. W. Misner, Mixmaster Universe, Phys. Rev. Lett. 22 (1969) 1071-1074

[27] D. Hobill, A. Burd, and A. Coley, Deterministic chaos in general relativity, Plenum Press, New York, 1994

[28] A. Ashtekar, M. Bojowald, and J. Lewandowski, Mathematical structure of loop quantum 
cosmology, Adv. Theor. Math. Phys. 7 (2003) 233-268, gr-qc/0304074

[29] T. Thiemann, QSD V: Quantum Gravity as the Natural Regulator of Matter Quantum Field Theories, Class. Quantum Grav. 15 (1998) 1281-1314, gr-qc/9705019

[30] M. Bojowald, Quantization ambiguities in isotropic quantum geometry, Class. Quantum Grav. 19 (2002) 5113-5130, gr-qc/0206053

[31] M. Bojowald, Loop Quantum Cosmology: Recent Progress, In Proceedings of the International Conference on Gravitation and Cosmology (ICGC 2004), Cochin, India. Pramana, 2004, gr-qc/0402053

[32] K. V. Kuchař, Time and interpretations of quantum gravity, In G. Kunstatter, D. E. Vincent, and J. G. Williams, editors, Proceedings of the 4th Canadian Conference on General Relativity and Relativistic Astrophysics, Singapore, 1992. World Scientific

[33] S. E. Rugh and B. J. T. Jones, Chaotic behaviour and oscillating three-volumes in Bianchi IX universes, Phys. Lett. A 147 (1990) 353-359

[34] P. Singh and A. Toporensky, Big Crunch Avoidance in k = 1 Loop Quantum Cosmology, Phys. Rev. D (2003) to appear, gr-qc/0312110

[35] M. Bojowald, J. E. Lidsey, D. J. Mulryne, P. Singh, and R. Tavakol, Inflationary Cosmology and Quantization Ambiguities in Semi-Classical Loop Quantum Gravity, gr-qc/0403106

[36] R. Penrose, Difficulties with inflationary cosmology, In E. Fenves, editor, Proceedings of the 14 th Texas Symposium on Relativistic Astrophysics, New York, 1990. N.Y. Acad. Sci.

[37] George F. R. Ellis, 83 years of general relativity and cosmology: progress and problems, Class. Quantum Grav. 16, (1999) A37-A75

[38] A. K. Raychaudhuri and B. Modak, Cosmological inflation with arbitrary initial conditions, Class. Quantum Grav. 5, (1988) 225-232

[39] I. Moss and V. Sahni, Anisotropy in the chaotic inflationary universe, Phys. Lett. B 178, (1986) 159-162 